\title{
Mitochondrial interactome quantitation reveals structural changes in metabolic machinery in failing murine heart
}

Arianne Caudal ${ }^{1,2 a}$, Xiaoting Tang ${ }^{3 a}$, Juan D. Chavez ${ }^{3}$, Andrew Keller ${ }^{3}$, Outi Villet ${ }^{2}$, Bo Zhou², Matthew A. Walker ${ }^{2}$, Rong Tian ${ }^{1,2 b^{*}}$ and James E. Bruce ${ }^{3 b^{*}}$

${ }^{1}$ Department of Biochemistry; ${ }^{2}$ Mitochondria and Metabolism Center, Department of Anesthesiology \& Pain Medicine; ${ }^{3}$ Department of Genome Sciences, University of Washington, Seattle, WA 98105, USA

${ }^{a}$ Co-first author; ${ }^{b}$ Co-corresponding author

${ }^{*}$ Correspondence to:

James E. Bruce, PhD

Rong Tian, MD, PhD

Department of Genome Sciences

Mitochondria and Metabolism Center

University of Washington

University of Washington

850 Republican Street

850 Republican Street

Seattle, WA 98109

Seattle, WA 98109

iimbruce@uw.edu

rongtian@uw.edu

Word count: 4231 


\begin{abstract}
Advancements of cross-linking mass spectrometry (XL-MS) for structural analysis of proteins bridges the gap between purified systems and native tissue environments. Here, isobaric quantitative protein interaction reporter technology (iqPIR) was utilized to further extend XL-MS to the first system-wide comparative study of mitochondrial proteins from healthy and diseased murine hearts. The failing heart interactome includes 602 statistically significant cross-linked peptide pairs altered in the disease condition. Structural insight into ketone oxidation metabolons, OXPHOS machinery, and nucleotide transporter hybrid-conformations, support mitochondrial remodeling in failing heart while bringing forth new hypotheses for pathological mechanisms. Application of quantitative cross-linking technology in tissue provides molecularlevel insight to complex biological systems difficult to model in cell culture, thus providing a valuable resource for study of human diseases.
\end{abstract}

Keywords: quantitative cross-linking; heart failure; cardiac hypertrophy; mitochondria; protein interactions; interactome; mass spectrometry; systems structural biology. 


\section{Introduction}

Mitochondria contain over 1,100 proteins that orchestrate diverse cellular functions, from intermediary metabolism and energy production to signaling and cell death (Pagliarini et al., 2008; Rath et al., 2020). Extensive genomic and proteomic approaches have resulted in a curated compendium of mitochondrial proteins with their sub-organellar localization (Rath et al., 2020). Advancements in cryo-electron microscopy, X-ray crystallography and NMR have greatly increased understanding of protein function and have enabled structure visualization of purified mitochondrial proteins and complexes with sizes unimaginable only a few years ago (Bridges et al., 2020; Gu et al., 2019; Qi et al., 2021; Spikes et al., 2020; Tucker and Park, 2019). However, within its native cellular or subcellular environment, protein function is dynamically-regulated by the presence of other proteins, post-translational modifications, substrates, cofactors, and transient conformational changes that are not directly measured with conventional techniques.

Chemical cross-linking combined with mass spectrometry (XL-MS) methods have emerged to provide structural insight from complex samples (Sinz, 2018). A cross-linker approach based on peptide synthesis chemistry to introduce MS cleavable features and affinity tags referred to as Protein Interaction Reporter (PIR) technology (Tang et al., 2005) has enabled interactomelevel structural studies with isolated functional mitochondria (Schweppe et al., 2017), intact virus particles (Chavez et al., 2012), live cells (Chavez et al., 2016; Weisbrod et al., 2013), and even whole tissue samples (Chavez et al., 2018), providing unique structural insights on protein interaction landscapes. While identification of in vivo protein-protein interactions (PPIs) and conformational features from cross-linked peptides increases knowledge about how proteins function within cells, quantitation of cross-linked peptide level changes during 
perturbation reveal molecular features that confer functional changes at the systems-level. For instance, quantitation of PIR cross-linked peptide levels in cells using SILAC (Ong et al., 2002) revealed functional differences relevant to acquired resistance to topoisomerase I inhibition therapy (Chavez et al., 2015). Moreover, quantitative PIR applications to cellular pharmacological studies revealed interactome changes that are drug-concentration dependent and mechanism-of-action specific (Chavez et al., 2019a; Chavez et al., 2016). Thus, quantitative cross-linking technologies enable visualization of interactome changes in living systems relevant to functional changes that could be informative in pathological comparisons. Recently, PIR technologies were further advanced to include isobaric quantitative capabilities that can enable quantitative interactome studies for systems without need for SILAC (Chavez et al., 2020a), as is employed here for failing heart mitochondrial interactome studies.

Due to the extraordinary energy requirements of the heart, cardiomyocytes contain the highest concentration of mitochondria of any cell in the body across mammalian species (Barth et al., 1992; Brown et al., 2017). Concurrently, mitochondrial dysfunction is a well-known maladaptive mechanism in the progression of heart failure. In this study, the feasibility of applying isobaric quantitative PIR (iqPIR) (Chavez et al., 2020a) XL-MS technology to mitochondria from healthy and failing mouse hearts is demonstrated. For the first time, integrated measurements of protein interactions, conformations, and surface accessibility allowing for comparative network analysis was enabled directly in tissue. These advancements provide the unique opportunity to study chronic, organ-level conditions, where cell modeling does not holistically recapitulate the bewildering complexity of disease. Of the total $\sim 3,800$ non-redundant cross-linked peptide pairs detected, $90 \%$ of cross-linked peptide pairs are quantified in at least one biological replicate. Statistical analysis revealed 602 cross-linked peptide pairs were significantly altered 
in failing hearts compared to sham controls, corresponding to altered catalytic pockets, hybrid conformations, and higher-order protein assemblies. This study provides molecular-level insight into basic mitochondrial function, and serves as a systems-level resource for generating new hypotheses for disease progression.

\section{Results}

\section{Quantitation of mitochondrial protein interactome in failing hearts}

A workflow was developed to assess mitochondrial protein interactome in failing hearts using iqPIR technology (Fig 1A). Heart failure was induced by transverse aortic constriction (TAC, $\mathrm{n}=6$ ) surgery in mice. Sham-operated (Sham) animals were used as controls $(n=6)$. TAC hearts demonstrated a decline in left ventricular fractional shortening (Fig 1B) and increased chamber size (Fig S1A-B) compared to Sham. At harvest, TAC mice showed significant cardiac hypertrophy (Fig 1C) and clear signs of pulmonary congestion (Fig S1C-D), indicating the development of heart failure.

Dissected TAC or Sham cardiac tissue was cross-linked with 2-plex iqPIR cross-linker with either Reporter Heavy (RH) or Stump Heavy (SH) iqPIR versions. TAC and Sham cross-linked samples were then mixed pairwise in a 1:1 ratio based on equal amount of total proteins. Thus, a total of 6 pairs of biological replicates were generated including 3 pairs of forward samples (RH labeled TAC combined with SH labeled sham) and 3 pairs of reverse samples (SH labeled TAC combined with $\mathrm{RH}$ labeled sham). The forward/reverse labeling strategy was used to evaluate if there was any quantitation bias caused by labeling direction. In total, 3,792 non-redundant cross-linked peptide pairs were identified from 6 pairs of TAC/Sham heart samples at an estimated FDR of $1 \%$ or less, corresponding to 2,734 lysine 
residue pairs and 507 protein pairs. The failing heart interactome network is available online in XLinkDB (Schweppe et al., 2016) (xlinkdb.gs.washington.edu, network table name = CaudalCellCommunity2021_Bruce). From the total coverage, over 90\% (3607/3792) of the cross-linked peptide pairs were quantified in at least one of 6 pairs of biological replicates. All quantified cross-link ratios were plotted against the p-value of each ratio (Figure 1D), which determined statistical outliers (significance threshold $p$-value $=2.5 \times 10^{-6}$ ). The data were further processed by applying a statistical t-test and Bonferroni correction to all quantified cross-linked peptides, then further filtered to a maximum of 2 missing ratios out of 6 pairs of biological replicates.

A final number of 602 cross-links were quantified showing statistically significant differences between TAC and Sham hearts (Supplemental Table 1). The heat map containing 602 crosslinks was generated using NG-CHM BUILDER (Broom et al., 2017) with Euclidean distance and complete linkage selected for hierarchical clustering (Figure 1E). A total of 316 crosslinked peptides were decreased while 286 cross-linked peptides were increased in TAC. iqPIR cross-linkers employ reactive esters to covalently react with surface accessible lysine residues. Dead-end (DE) labeled peptides are generated when only one ester reacts with a lysine residue, while the other ester is hydrolyzed. This feature is useful as a read-out for the surface accessibility of proteins, and an indirect survey of relative protein level changes. Since PPI levels can be affected by endogenous protein levels, DE-labeled peptide quantitation and intra-cross-linked peptides were used to estimate protein abundance for each cross-linked peptide pair as demonstrated previously by Chavez et al (Chavez et al., 2020a). For each non-redundant cross-link pair, the mean Log2 ratios for Protein A and Protein B were calculated from all DE peptides (Supplemental Table 1, Fig S1E). In addition, reproducibility of 
the data was evaluated by applying linear regression to 6 pairs of biological replicates on a pair-wise basis. R-squared values were calculated and shown in Figure 1F. Despite the known individual variations in the TAC model, strikingly high data correlation and consistency were observed across all 15 comparisons of 6 TAC/Sham pairs. It is worth noting that each sample is 1:1 mix of cross-linked proteins from two different animals thus regression fitting of two samples involved 4 different animals. The correlations of forward and forward labeling samples (average $\mathrm{R}^{2}=0.54$ ) are similar to those of forward and reverse labeling samples (average $\mathrm{R}^{2}=0.45$ ) (Supplemental Table 2), which further confirmed the consistency and reproducibility of iqPIR quantitation results. The subset of cross-linked peptide pairs displaying statistically different ratios in TAC were further analyzed by interaction network analysis (Fig S1F). Comprehensive views of pathways and proteins exhibiting large statistical changes (Log 2 ratios are generally $\geq 1$ or $\leq-1$ ) led to key highlights of TAC-induced structural changes that will be further discussed in greater detail in the following sections.

\section{Active conformational states of ketone oxidation proteins enriched in TAC}

Recent studies demonstrated an increase in ketone consumption by failing hearts (Aubert et al., 2016; Bedi et al., 2016; Murashige et al., 2020). Enzymes for ketone oxidation, D- $\beta$ hydroxybutyrate dehydrogenase $(\mathrm{BDH})$ and Succinyl-CoA:3-oxoacid-CoA transferase (SCOT1), were upregulated in the failing heart but mechanisms driving the flux of ketone oxidation were not fully understood (Aubert et al., 2016; Bedi et al., 2016; Kolwicz et al., 2016). Circulating ketones, primarily generated by the liver, are taken up by cardiomyocytes and metabolized via three consecutive reactions catalyzed by BDH, SCOT1 and Acetyl-CoA acetyltransferase (THIL) to produce acetyl-CoA for further oxidation in the TCA cycle (Fig 2A). 
Consistent with increased expression of ketone metabolism proteins in failing myocardium (Aubert et al., 2016; Bedi et al., 2016), DE quantitation of BDH, SCOT1 and THIL were increased in the iqPIR dataset (Supplemental Table 1). However, quantification of cross-linked lysine residues in SCOT1 and THIL did not match the changes of DE suggesting the presence of protein structural changes beyond changes to abundance (Fig 2B).

SCOT1, encoded by the Oxct1 gene, is a tetramer of two inter-connected homodimers (Fig 2C). It transfers a Coenzyme $A(C o A)$ moiety from succinyl-CoA to acetoacetate to form acetoacetyl-CoA and succinate (Fig 2A). The reaction involves E344 in the active site, which sits between two binding pockets for succinate/acetoacetate (residues 321-329, Loop A) and CoA (residues 374-386, Loop B), respectively (Tammam et al., 2007). Four non-redundant cross-linked peptide pairs in SCOT1 were increased during TAC (Fig 2B, S2A). Cross-linked peptides containing K286, K296, K418, and K421 were mapped onto the SCOT1 structure (human SCOT1 PDB: 3DLX) and pinpointed a key surface near Loop A (Fig 2C). During catalysis, the C-terminal undergoes a 17 degree angle domain rotation where Loop A must conform in order to alleviate steric clashing with the static N-terminal (Tammam et al., 2007). We hypothesize that the rotation renders the cross-linking of the four peptide pairs possible. Thus, increases in the cross-linked peptide pairs suggest a greater fraction of SCOT1 assumes an active conformational state in TAC. As K286 and K296 reside in a relatively disordered region of SCOT1, where detailed structural information is unavailable. However, large conformational change in the C-terminal domain, where the four cross-linked peptide pairs reside, is observed when apo (PDB: 30XO, chain A) and substrate-bound (PDB: 30XO, chain E) porcine SCOT1 monomers are aligned (Fig S2B) (Coker et al., 2010; Fraser et al., 2010) supporting the notion that active enzyme assumes distinct conformation in the region. In 
humans, naturally occurring mutations in Loop A, such as G324E and L327P, result in SCOT1 loss-of-function (Shafqat et al., 2013), indicating the importance of flexible conformations observed in this region.

The homodimer link between SCOT1 K296-K296 increased 2-fold in TAC ( $p$-value=2.13E-13) indicating increased interaction between two monomeric units of SCOT1 (Fig 2A). However, the K296-K296 distance mapped onto the human tetramer structure (PDB:3DLX) (Figure S2C) is $66 \AA$, which exceeds the span possible by iqPIR cross-linkers (Ca-Ca<42A). These results suggested that SCOT1 exists in an alternative conformation or higher order assembly which is increased in TAC. To determine if this is the case, human SCOT1 tetramers were subjected to molecular docking (Schneidman-Duhovny et al., 2005). With no distant constraints, docking results from 6 of the top 10 solutions (Fig 2D, S3A) consistently placed this region at the interface between two tetramers. The $\mathrm{N}$-terminal domain is relatively static (Fig S2B) during catalysis, yet the cross-linked peptide pair K214-K274 exhibited decreasing quantitation during TAC (Fig 2C). Higher-order docking solutions of SCOT1 place K214 and K274 buried at the oligomeric interface between two tetramers in three solutions (Fig S3B). In these conformations, either K214 or K274 would be inaccessible to cross-linking, which further supports possible enrichment of SCOT1 oligomer in TAC.

In contrast to SCOT1, the cross-link between K242-K260 in THIL decreased in TAC despite a moderate increase of DE (mean Log2 ratio=0.19, Fig $2 \mathrm{~B}$ ). This peptide pair was mapped to lysine residues located on adjacent monomers of the human crystal structure (Fig 2E, Substrate-bound THIL PDB 2IBW). Residue K260 (conserved human K263) helps form the CoA binding pocket and provides the only salt-bridge contact with the 3'phosphate moiety (Haapalainen et al., 2007). Post-translational modification (PTM) at either K242 or K260 
provides one possible explanation for decreased cross-linking. However, previous reports demonstrated that hyperacylation at K260 suppressed THIL activity (Dittenhafer-Reed et al., 2015; Still et al., 2013) Increased ketone oxidation, as observed in heart failure (Aubert et al., 2016; Bedi et al., 2016; Murashige et al., 2020), would likely increase salt-bridge occupancy by forming the K260-CoA interaction necessary for catalysis and consequently, decrease crosslinking accessibility (Figure S3C).

Taken together, quantitative iqPIR analysis confirmed the upregulation of ketone metabolism proteins as previously reported, and furthermore, suggest possible mechanisms for active protein conformations in the failing hearts. These findings provide greater insight on how ketone metabolism is potentially adapted in the failing heart that could enable future therapeutic developments.

\section{Decreased interaction between NDUA4 and C6XB1 affects CIV activity in TAC}

Mitochondrial respiration, assessed by oxygen consumption rate (OCR), was reduced in TAC hearts (Fig 3A-B). Multiple mechanisms have been proposed for impaired OCR, among which altered function of electron transport chain (ETC) has been proposed (Brown et al., 2017). Quantitative mitochondrial interactome data analysis revealed five cross-linked lysine pairs between NDUA4 and CX6B1 (human COX6B isoform 1) in Complex IV that were significantly decreased in TAC (Fig 3C, Supplemental Table 3). Complex IV (Cytochrome C oxidase, CIV) is the terminal end of the electron transport chain, which accepts two electrons from Cytochrome C (CYC) to reduce oxygen into water. Due to its high sensitivity to detergent conditions (Balsa et al., 2012; Zong et al., 2018) interaction between NDUA4 and other subunits of Complex IV has been difficult to assess. Since iqPIR cross-linkers were applied 
directly to heart tissue to secure PPIs with covalent bonds, the disruption of detergent-sensitive interactions such as those of NDUA4 was avoided with this approach. Identification of multiple NDUA4 inter-protein cross-linked peptide pairs provides direct and definitive evidence that NDUA4 exists as a component of CIV in cardiac mitochondria and provides insight into the NDUA4-CX6B1 interaction. Cross-linked peptides containing residues $\mathrm{K} 10, \mathrm{~K} 13$, and $\mathrm{K} 85$ of CX6B1 and K56, K74 and K76 of NDUA4 were mapped to disordered regions of both proteins residing in the IMS (Fig 3D, PDB: 5Z62) making further structural analysis challenging. Mutations in CX6B1 R20 which disrupt a salt-bridge with D17 (Massa et al., 2008) are hypothesized to cause instability of CIV leading to encephalomyopathy and hypertrophic cardiomyopathy in patients (Abdulhag et al., 2015). R20 is likely stabilized by a salt-bridge with nearby NDUA4 D60, although this side-chain is only partially resolved in the structure (Figure S4A, PDB: 5Z62). However, Complex IV activity was found to be significantly decreased in TAC (Figure S4B) raising the possibility that NDUA4-CX6B1 interaction modulated CIV function.

Furthermore, as NDUA4 blocks the CIV dimerization interface, its presence maintains CIV primarily as a monomer thus facilitating Complex I-III $-\mathrm{IV}$ respirasome assembly (Zong et al., 2018). Alterations in the interaction between NDUA4 and other subunits of CIV could therefore affect respirasome formation. Decreased $\mathrm{Cl}-\mathrm{ClII}_{2}$-CIV respirasome has been previously reported in heart failure (Rosca et al., 2008). Thus, quantitative mitochondrial interactome analysis reveals remodeling of Complex IV structure as a new contributor to impaired mitochondrial respiration within the failing heart.

\section{Enrichment of an intermediate state of ADPIATP carrier detected in TAC}


Exchange of ATP and ADP between mitochondria and cytosol is achieved by the mitochondrial ADP/ATP carrier (ADT), a highly conserved, abundant, and extensively studied translocase (Pebay-Peyroula et al., 2003). ADT is a dual gated transporter that interconverts between at least two known distinct structural conformations (Fig 4A). During a normal cycle, the cytoplasm-open state (C-state) faces the IMS and the gate to the mitochondrial matrix is closed via a series of salt-bridges, allowing for the release of a bound ATP and the binding of a new cytosolic ADP (Pebay-Peyroula et al., 2003). The binding of ADP induces a conformational shift to the matrix-open state (M-state) in which the gate to the IMS is closed by formation of new salt-bridges and a hydrophobic plug (Ruprecht et al., 2019). The M-state releases cytosolic ADP in exchange for a newly charged ATP and the cycle repeats. These conformational changes permit the transport of large nucleotide solutes across small protein carriers while preventing proton leak throughout the exchange process (Karch et al., 2019). Mammalian ADT exists in four isoforms which exhibit tissue-dependent but overlapping expression patterns.

Quantitative iqPIR analysis revealed increased levels of seven non-redundant cross-linked peptide pairs of ADT1 in TAC (Fig 4B, S4C). It should be noted that other ADT isoforms and multimeric ADT structures may also be present that could explain increases in these observed links (Figure S4D), but ADT1 is the predominant isoform in cardiac and skeletal muscle (Bround et al., 2020). Cross-links between K23-K92, K23-K94, K23-K96 and K23-K199 are compatible with the C-state (Pebay-Peyroula et al., 2003) of ADT1 because all lysine residues are solvent exposed in this conformation (Fig 4C, S4B, bovine ADT1 PDB: 1OKC,

Supplemental Video 1). However, these links are not compatible with the M-state because $\mathrm{K} 92, \mathrm{~K} 94, \mathrm{~K} 96$, and $\mathrm{K} 199$ must penetrate occluded volume of the protein in this conformation. 
Moreover, K96 and K199 form salt bridge interactions with D196 and D292 that contribute to the ADT1 gate closure to the IMS (Fig 4D, S4E, TtAac ADT1 PDB: 6GCI, Supplemental Video 1). Although the $\mathrm{K} 23$ and $\mathrm{K} 33$ cross-link is compatible with distance constraints, it is known that K33 partakes in the hydrogen-bond network needed to stabilize the C-state by forming a salt-bridge with D232 (Pebay-Peyroula et al., 2003) (Fig S4F). On the other hand, cross-links between K23-K33, K23-K147, and K33-K147 are compatible only with the M-state (Fig 4D, S4D). Thus, increases of all seven links would indicate that both C-state and M-state have increased in TAC. This is, however, unlikely given the mutual exclusivity of M- and C-states and the observation of no large change in ADT1 protein levels (Log2 TAC/Sham= 0.5). Functionally, increased enrichment of C-state in failing hearts, in which ATP production is known to be impaired, is also counter-intuitive. Collectively, increases in these seven crosslinked peptide pairs and ADT1 protein level measurements in TAC hearts cannot be explained by either a shift between C-state and M-state of ADT1 in heart failure.

Perhaps a more likely explanation is the existence of an alternative state of ADT1, with which all quantified cross-links are compatible, and this new conformation has increased in TAC. To satisfy all the cross-links in one single state, lysine residues involved in gating at either C- or M-state must be accessible to cross-linkers. This new conformation could be consistent with the P-state which was recently proposed to be simultaneously open to both the IMS and the matrix, synonymous with non-selective mPTP conductance (Karch et al., 2019; Ruprecht et al., 2019). Although there is currently no known structural evidence, the non-selective P-state is hypothesized to provide an open channel where the lysine residues responsible for gating are not involved in salt bridge formation and thus, may be available for cross-linking. Thus, our results provide novel structural evidence that heart failure is associated with increased 
conformational enrichment of ADT1 that is non-functional for ADP/ATP translocation but likely possesses non-selective conductivity (Bertholet et al., 2019).

\section{Discussion}

In this study, iqPIR technology achieved one of the highest levels of cross-linking complexity resolved from tissue thus far. Our comparative studies of mitochondria in normal and failing hearts yield quantitative changes in 98 mitochondrial proteins, providing a system's view of protein structure remodeling in heart failure. Among the 602 cross-linked peptide pairs that showed statistically significant level differences in TAC vs. Sham hearts, enrichment of active ketone oxidation enzymes, altered interactions among Complex IV subunits and novel conformational enrichment of the ADP/ATP transporter were revealed in TAC hearts. These findings advance mitochondrial and heart failure research in several ways. First, the data elucidate new structural information on key players in mitochondrial function, providing a basis for developing future therapies. Second, the results enable generation of novel hypotheses for mechanisms of mitochondrial maladaptation in disease. Most importantly, the quantitative interactome dataset provides a valuable resource for exploration and visualization of changes in many other proteins not discussed here that may be important for improved understanding of mitochondrial function and heart failure pathology.

Using iqPIR technology as a discovery-based approach in this study, conformational and interaction changes in mitochondrial proteins and complexes were observed that possess direct functional relevance to metabolic changes in heart failure. Identification of SCOT1 oligomer metabolons and increased active conformational states of SCOT1 and THIL in the present study are corroborated by previously reported metabolic changes in failing hearts. 
Therefore, this agreement serves as affirmation of the utility of quantitative in vivo cross-linking and mass spectrometry as a means to gain greater molecular-level insight on changes in heart failure. Importantly, changes in ketone metabolism has been thus far attributed to substrate and enzyme abundance in heart failure with no information on regulatory mechanisms (Kolwicz et al., 2016). Our results provide new insight into metabolic remodeling, suggesting that active conformations exist beyond mere increases in protein levels.

The first direct evidence for the NDUA4 existence as a CIV subunit within cardiac tissue was provided by PIR technology (Chavez et al., 2018). The present iqPIR data further confirms that interaction and enables the first quantitation of the NDUA-CX6B1 interaction in failing and control heart tissues with five non-redundant Lys-Lys residue pairs between NDUA4 and CX6B1. The decrease in NDUA4-CX6B1 interactions and concomittant decrease in CIV activity in heart failure samples suggests this interaction could serve as a potential modulator of CIV activity. NDUA4 is thought to maintain CIV monomeric population by occupying the CIV dimerization interface. Decreased NDUA4-CX6B1 cross-links, coincident with decreased CIV activity in TAC, supports the hypothesis that monomer and respirasome populations are functionally active populations (Zong et al., 2018). It remains unclear whether destabilization of NDUA4 during TAC results in consequential alterations of CIV dimeric pools, and what role this population, if any, may have in disease pathology (Ramzan et al., 2019). Nonetheless, the present observation that NDUA4-CX6B1 interactions decrease in heart failure presents a new, previously unrecognized target for future studies and possible therapies that stabilize this interaction as treatment to restore or prevent CIV functional decline in heart failure. Mitochondrial ADP/ATP carrier (ADT) has long been proposed to contribute a pore-forming component of the mitochondrial permeability transition pore (mPTP) (Karch et al., 2019; 
Kokoszka et al., 2004). However, several decades of genetics and physiological studies have yielded ambiguous evidence. Transient opening of mPTP has physiological function such as regulating mitochondrial calcium homeostasis or reactive oxygen species signaling while prolonged mPTP contributes to cell death under stress conditions including heart failure (Bround et al., 2020). Our results provide critical structural information consistent with recently proposed P state of ADT which opens to both matrix and inter-membrane space (Bround et al., 2020). It remains unclear whether the P-state is static, or is resultant from rapid interconversion between two incompletely gated structures. Nonetheless, increased presence of such a state compromises nucleotide translocation function and increases mitochondrial proton leaks leading to impaired energetics. In support of this notion, a mitochondrial-targeted tetrapeptide, elamipretide (SS-31), is shown to bind K23 of ADT1 and reduces proton leak in mitochondria of aging animals (Chavez et al., 2020b). Thus, changes in the conformational state of ADT warrants further investigation both as a disease mechanism and a therapeutic target.

In summary, we demonstrate that iqPIR and mass spectrometry constitute a powerful technology to quantify protein structural changes at the systems-level in tissues. Application of this technology to animal models of heart failure generated a new mitochondrial protein interactome that revealed novel mechanisms that underpin potential therapies.

\section{Acknowledgments}

We thank all members of the Tian and Bruce labs their thoughtful discussion and support. We thank the University of Washington Proteomics Resource for advice and helpful discussions. 
We thank Dr. Yun-Wei A. Hsu for assistance with the animal models. We thank Dr. Julia Ritterhoff and Dr. Frauke Drees for technical support and guidance.

\section{Sources of funding}

This work was supported in part by U.S. National Institutes of Health (NIH) grants HL110349, HL129510, HL142628 (to R.T.), HL144778, GM097112, R35GM136255 (to J.E.B.), American Heart Association Predoctoral Fellowship 20PRE35120126 (to A.C.), AHA Postdoctoral Fellowship 18POST33990352 (to B.Z.), and NIH 2T32DK007247-41 to (M.A.W).

\section{Contributions}

A.C., X.T., J.D.C., R.T., and J.E.B designed the experiments. A.C., X.T., J.D.C, A.K, M.A.W, R.T., and J.E.B. wrote the manuscript. A.C, X.T, R.T, and J.E.B edited the manuscript. A.C., X.T., J.D.C., A.K., performed formal analysis. A.C., B.Z., M.A.W performed animal experiments. A.C. and J.D.C., performed cross-linking experiments. J.D.C performed protein preparation. J.D.C, X.T., and A.K performed mass spectrometry raw data acquisition and processing. A.K. developed computational tools to support structural protein analysis and cross-linking quantitation. O.V. performed TAC surgery. R.T and J.E.B. supervised the project.

\section{Declaration of interests}

None declared. 


\section{Figure 1}

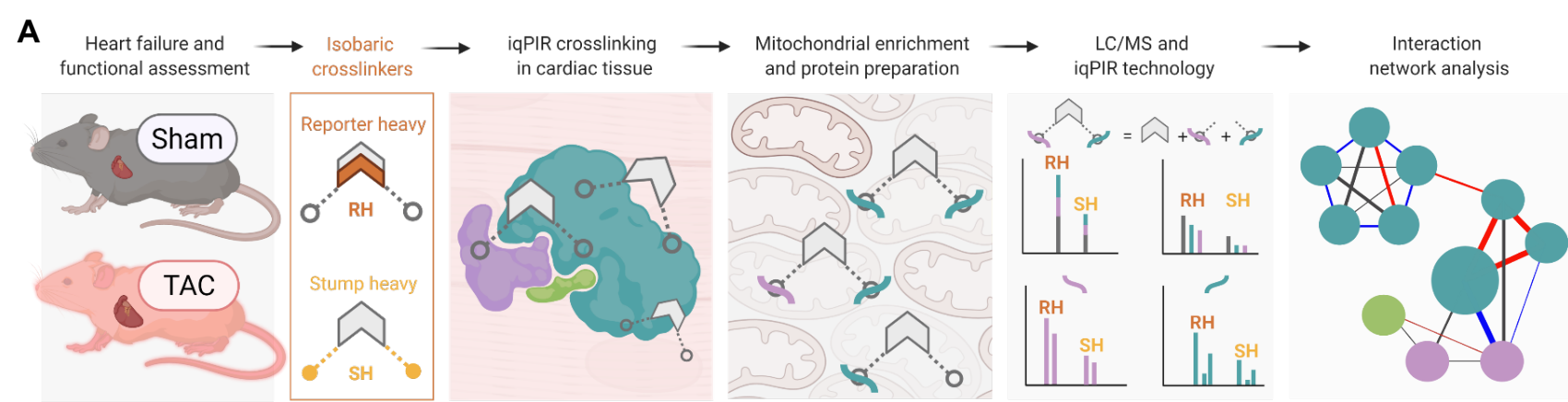

B

Fractional
Shortening

(\%)

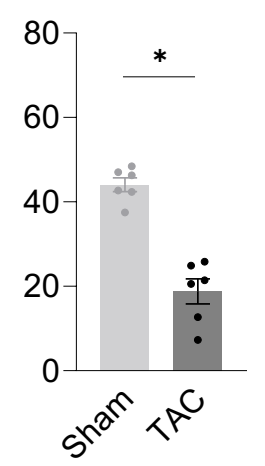

C
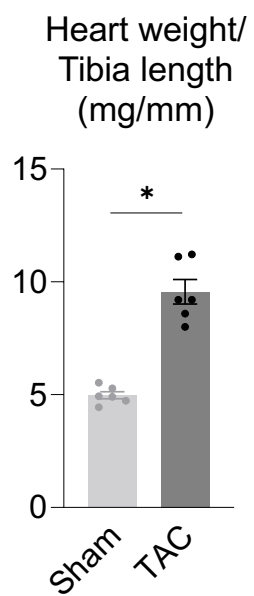

E

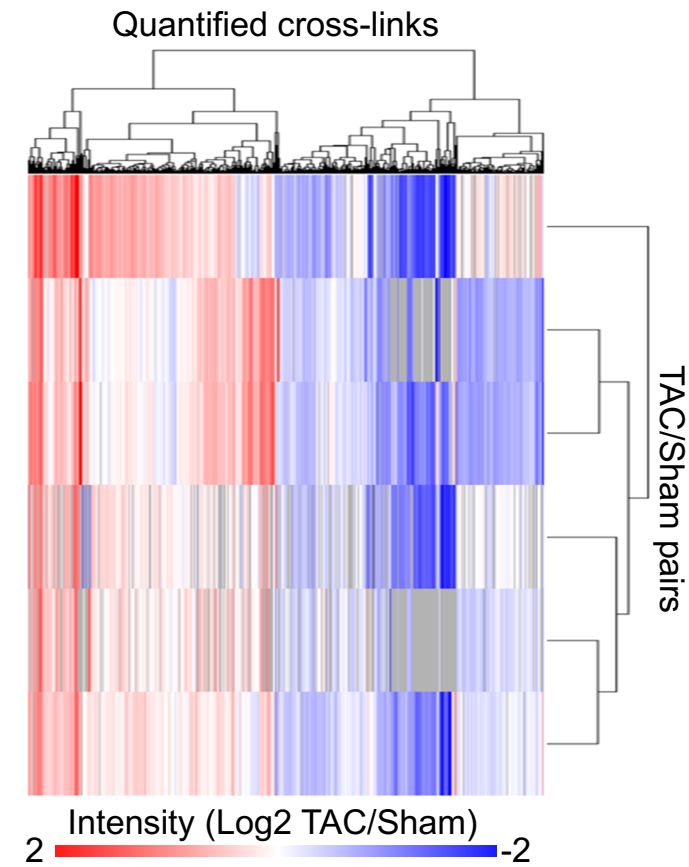

D

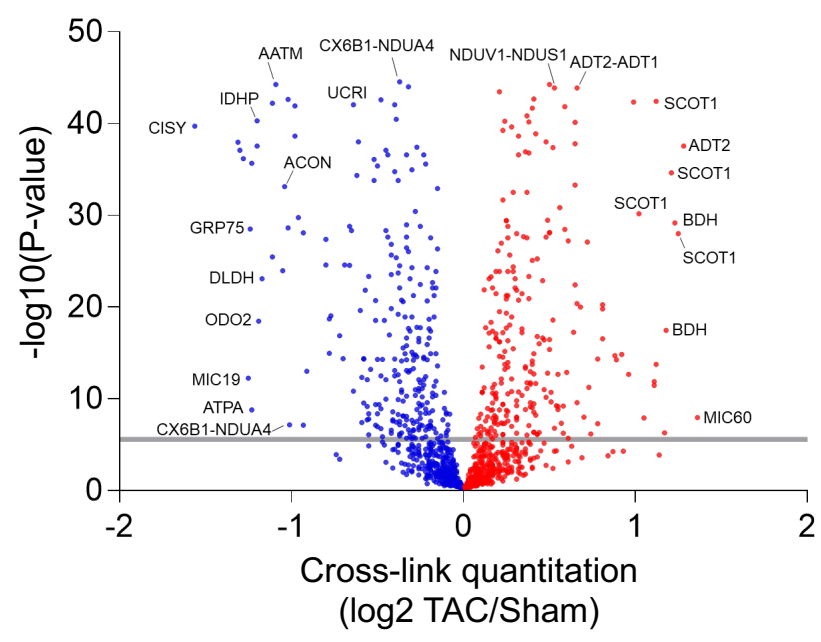

F

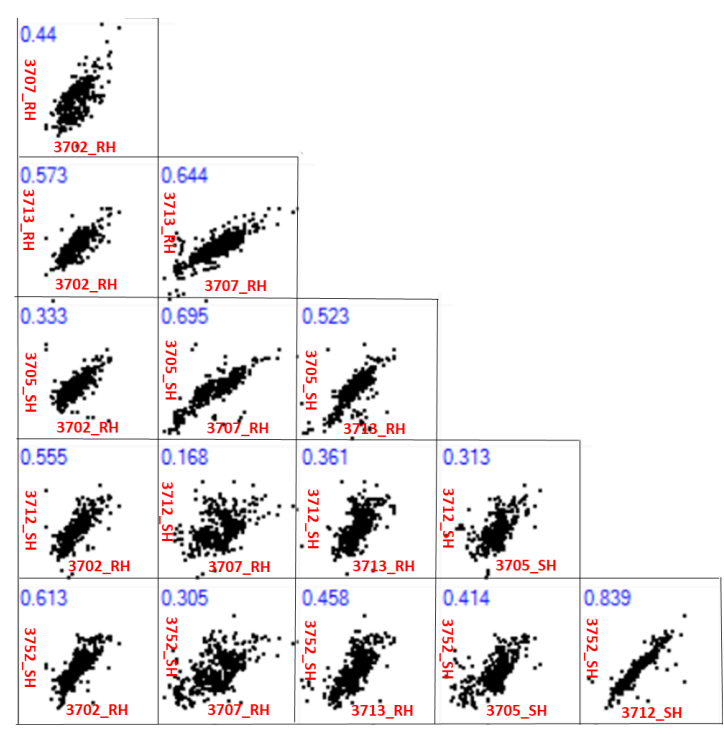




\section{Figure 1: Quantitation of mitochondrial protein interactome in failing hearts}

(A) Schematic of quantitative failing heart interactome pipeline. Briefly, TAC and Sham hearts were excised and subjected to stump-heavy and reporter-heavy iqPIR cross-linking reaction followed by mitochondrial enrichment. Samples were pooled for downstream processing and data acquisition by LC/MS. Identical cross-linked peptide pairs from TAC and Sham samples have identical masses yet produce distinct quantitative isotope signatures in $\mathrm{MS}^{2}$ spectra, and their intensities enable relative quantitation of cross-linked peptides. Illustration created with BioRender.com.

(B) Assessment of cardiac function measured by fractional shortening (\%) using echocardiography in TAC and Sham groups four-weeks post-surgery.

(C) Cardiac hypertrophy measured by ratio of heart weight $(\mathrm{mg})$ to tibia length $(\mathrm{mm})$ harvest.

(D) Volcano plot of quantified cross-link lysine ratios (Log2 TAC/Sham) versus statistical significance. Significance threshold set to $p=2.5 \times 10-6(-\log 10=5.6)$. Blue circles indicate crosslinked peptide ratios with decreasing quantitation, while red circles indicate cross-linked peptide ratios with increasing quantitation (relative to TAC).

(E) Heat map illustration of quantified cross-linked peptide pairs showing significant changes between TAC and Sham groups, filtered for values present in at least 4/6 biological replicates.

(F) Pair-wise scatter plots of 6 pairs of biological replicates. Correlation of Log2 ratios of two biological replicates as shown in $\mathrm{X}$-axis and $\mathrm{Y}$-axis was evaluated with linear regression. $\mathrm{R}$ squared value marked in blue font on top left of each figure.

For (B-C), all data are $n=6, A V G+/-S E M,{ }^{*}$ denotes $p<0.05$ by Student's $t$ test. 
bioRxiv preprint doi: https://doi.org/10.1101/2021.08.13.456027; this version posted August 14, 2021. The copyright holder for this preprint (which was not certified by peer review) is the author/funder. All rights reserved. No reuse allowed without permission.

\section{Figure 2}

A

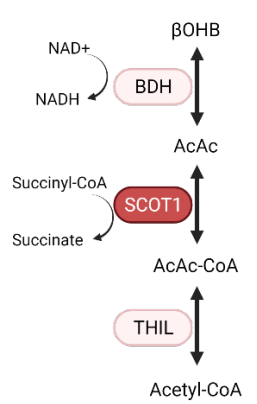

C

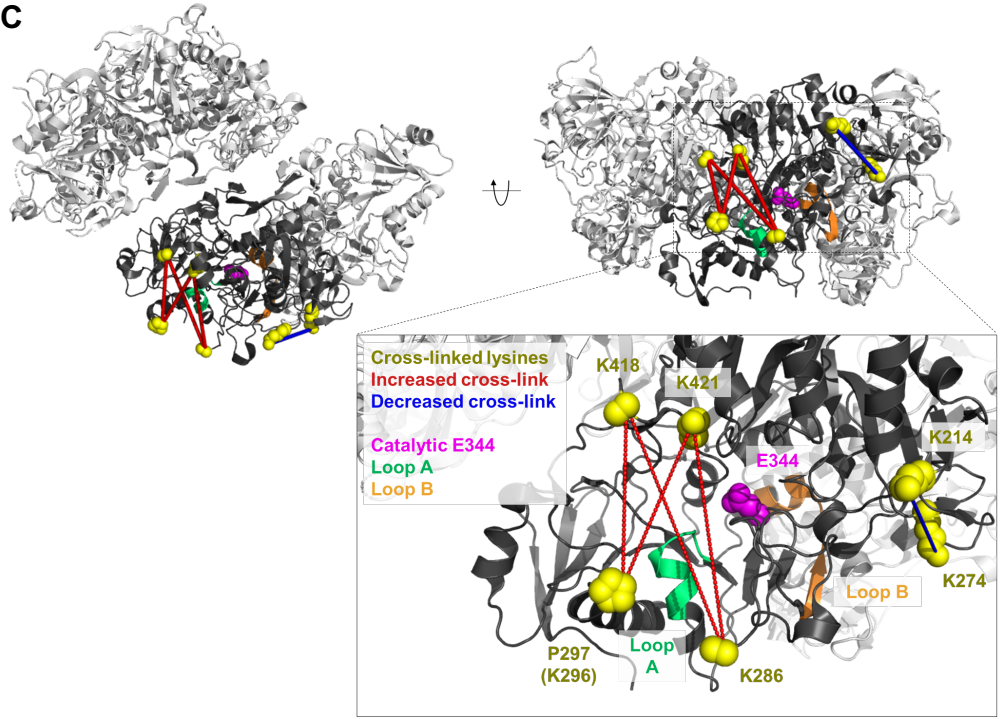

B

\begin{tabular}{|c|c|c|c|c|}
\hline \multicolumn{2}{|c|}{ Cross-linked peptide pair } & \multicolumn{2}{c|}{$\begin{array}{c}\text { Log2 ratio } \\
\text { (TAC/Sham) }\end{array}$} \\
\hline Protein & LysA & LysB & $\begin{array}{c}\text { Mean } \\
\text { XL }\end{array}$ & $\begin{array}{c}\text { Mean } \\
\text { DE }\end{array}$ \\
\hline SCOT1 & 296 & 421 & 1.25 & 0.87 \\
\hline SCOT1 & 286 & 418 & 1.02 & 0.87 \\
\hline SCOT1 & 296 & 418 & $\mathbf{0 . 9 9}$ & 0.87 \\
\hline SCOT1 & 286 & 421 & 1.12 & 0.87 \\
\hline SCOT1 & 296 & 296 & 1.05 & 0.87 \\
\hline SCOT1 & 214 & 274 & -1.69 & 0.87 \\
\hline THIL & 260 & 242 & -1.47 & 0.19 \\
\hline
\end{tabular}

D
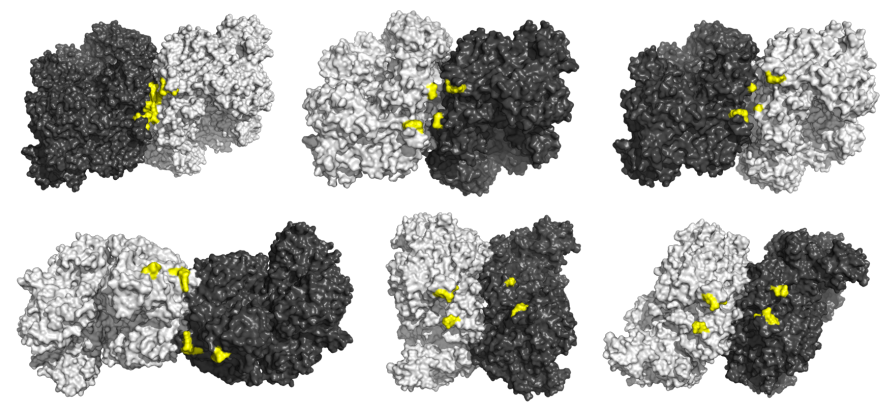

E

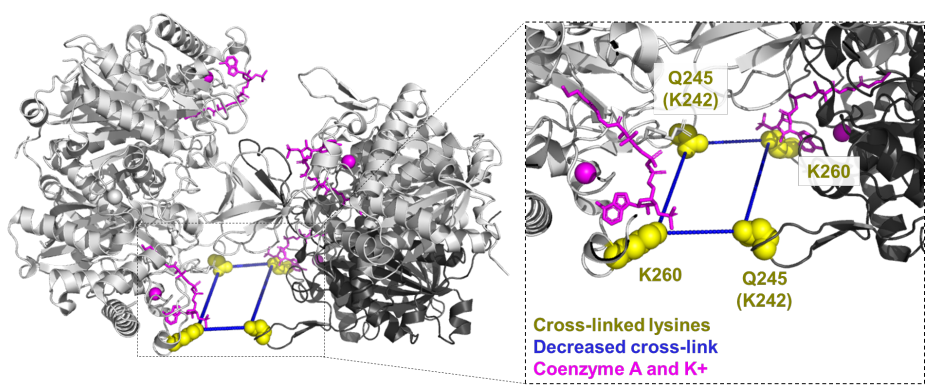




\section{Figure 2: Active conformational states of ketone oxidation proteins enriched in TAC}

(A) Schematic of ketone oxidation pathway.

(B) Table summarizing the mean cross-linking ratio and DE ratio for cross-linked peptide pairs exhibiting quantitative changes in ketone oxidation machinery.

(C) Human SCOT1 tetrameric structure (PDB: 3DLX) shown in grey. Monomeric unit is shown in black centered around the catalytic E344 (magenta). Cross-linked lysine side-chains (K286, K296/P297, K418, and K421, shown as yellow spheres) surround Loop A and together form four cross-links found to increase in TAC. Loop A (CoA binding site, residues 321-329, chain shown in green) is a dynamic region that must undergo conformational change to prevent steric clashing of $\mathrm{N}$ - and $\mathrm{C}$-termini during catalysis. Decreasing cross-linked peptide pair, K214-K274, is also shown (yellow spheres) near Loop B (Succinate/Acetoacetate binding site, residues 374-386 chain shown in orange). P297 is shown due to missing density of K296.

(D) Six solutions (from top ten highest scoring) from molecular docking of two human SCOT1 tetramers (grey and black, PDB: 3DLX) using Symmdock (Schneidman-Duhovny et al., 2005) indicative of higher order oligomerization. Residues surrounding K296 (missing density) are shown in yellow, and are consistently docked in close proximity $(\mathrm{C} \alpha-\mathrm{C} \alpha<42 \AA)$ to each other, providing evidence for K296-K296 cross-link being between two SCOT1 tetramers.

(E) Human THIL (PDB: 2IBW) tetrameric structure with bound CoA and $\mathrm{KCl}$ (magenta) highlighting the decreased cross-link between K260-K242/Q245 (sidechains shown as yellow spheres). K260 forms the only direct contact with CoA. K242 resides in the anionic loop meant to capture incoming substrate. Mouse K242 is equivalent to human Q245. 


\section{Figure 3}
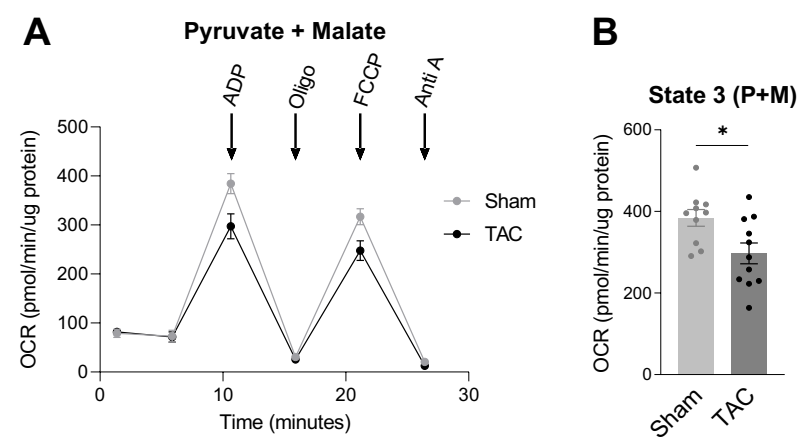

C

\begin{tabular}{|c|c|c|c|c|c|c|}
\hline \multicolumn{4}{|c|}{ Cross-linked peptide pair } & \multicolumn{3}{|c|}{ Log 2 ratio (TAC/Sham) } \\
\hline ProteinA & ProteinB & LysA & LysB & $\begin{array}{c}\text { Mean } \\
\mathrm{XL}\end{array}$ & $\begin{array}{c}\text { Mean DE } \\
A\end{array}$ & $\begin{array}{c}\text { Mean DE } \\
B\end{array}$ \\
\hline CX6B1 & NDUA4 & 85 & 56 & -1.01 & -0.41 & -0.27 \\
\hline CX6B1 & NDUA4 & 10 & 74 & -0.55 & -0.41 & -0.27 \\
\hline CX6B1 & NDUA4 & 10 & 76 & -0.49 & -0.41 & -0.27 \\
\hline CX6B1 & NDUA4 & 13 & 74 & -0.49 & -0.41 & -0.27 \\
\hline CX6B1 & NDUA4 & 13 & 76 & -0.44 & -0.41 & -0.27 \\
\hline
\end{tabular}

D

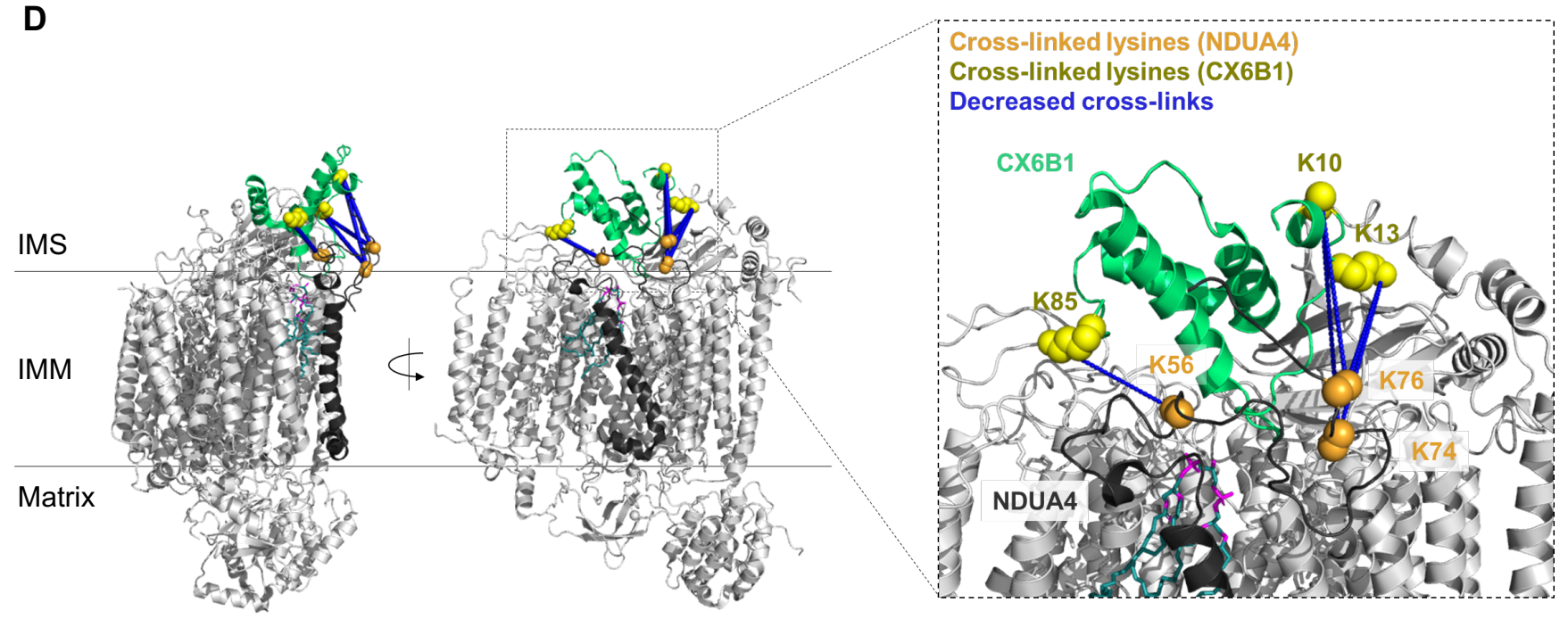

Figure 3: Decreased interaction between NDUA4 and C6XB1 affects CIV activity in TAC

(A) Oxygen consumption rate of mitochondria isolated from TAC and Sham hearts given

pyruvate/malate substrates followed by sequential injections of ADP, Oligomycin, FCCP, and

Antimycin A, measured by Seahorse XFe24 Analyzer.

(B) State III-driven respiration of isolated mitochondria (after addition of ADP).

(C) Table summarizing the mean cross-linking ratio and DE level ratio for cross-linked peptide pairs exhibiting quantitative changes in NDUA4 and CX6B1 subunits of CIV. 
(D) Monomeric Complex IV (grey, PDB: 5Z62). Interactions between NDUA4 (black) and

CX6B1 (green) subunits are shown. Cross-linked lysine sidechains are shown as yellow (CX6B1) or orange (NDUA4) spheres. Cross-links exhibit a structural scaffold on the IMSfacing interface of CIV, which are decreased in TAC. Cardiolipin bound to CIV is shown in teal. For (A-B), all data are $n=10-11, A V G+/-S E M$, *denotes $p<0.05$ by Student's $t$ test. 
bioRxiv preprint doi: https://doi.org/10.1101/2021.08.13.456027; this version posted August 14,2021 . The copyright holder for this preprint (which was not certified by peer review) is the author/funder. All rights reserved. No reuse allowed without permission.

\section{Figure 4}

A

C-State M-State C-State

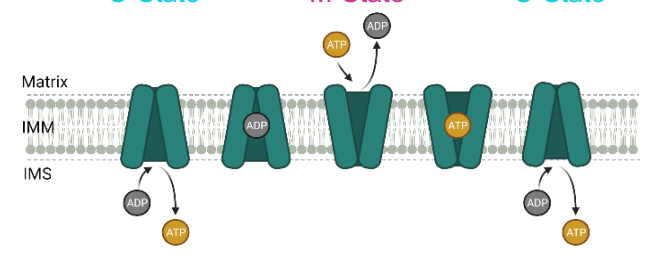

B

\begin{tabular}{|c|c|c|c|c|c|c|c|c|}
\hline \multicolumn{3}{|c|}{ Cross-linked peptide pair } & \multicolumn{2}{|c|}{$\begin{array}{l}\text { Log2 ratio } \\
\text { (TAC/Sham) }\end{array}$} & \multicolumn{2}{|c|}{ C-state } & \multicolumn{2}{|c|}{ M-state } \\
\hline Protein & LysA & LysB & $\begin{array}{c}\text { Mean } \\
\mathrm{XL}\end{array}$ & $\begin{array}{c}\text { Mean } \\
\mathrm{DE}\end{array}$ & $\begin{array}{c}\text { Euclidean } \\
\text { distance }\end{array}$ & $\begin{array}{l}\text { Jwalk } \\
\text { SASD }\end{array}$ & $\begin{array}{c}\text { Euclidean } \\
\text { distance }\end{array}$ & $\begin{array}{l}\text { Jwalk } \\
\text { SASD }\end{array}$ \\
\hline ADT1 & 92 & 23 & 0.74 & 0.5 & 22.6 & 23.78 & 20.08 & 99 \\
\hline ADT1 & 94 & 23 & 0.55 & 0.5 & 28.13 & 31.94 & 24.1 & 61.38 \\
\hline ADT1 & 96 & 23 & 0.79 & 0.5 & 27.32 & 29.61 & 25.47 & 99 \\
\hline ADT1 & 199 & 23 & 0.65 & 0.5 & .96 & 32.09 & 22.62 & 99 \\
\hline ADT1 & 33 & 23 & 03 & 05 & 3.52 & 16.64 & 14.41 & 17.78 \\
\hline ADT1 & 147 & 23 & 0.65 & 0.5 & 23.53 & 66.86 & 27.25 & 30.92 \\
\hline ADT1 & 147 & 33 & 0.65 & 0.5 & 19.12 & 99 & 24.51 & 31.65 \\
\hline
\end{tabular}

C

$$
\text { C-State }
$$

D

M-State

IMS
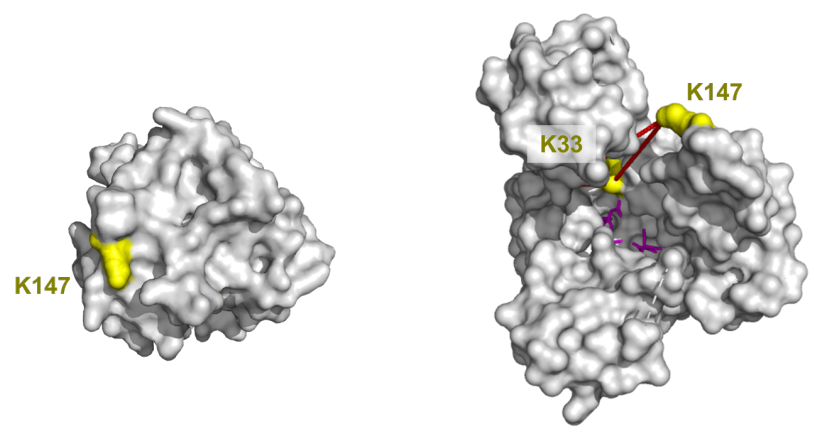

1
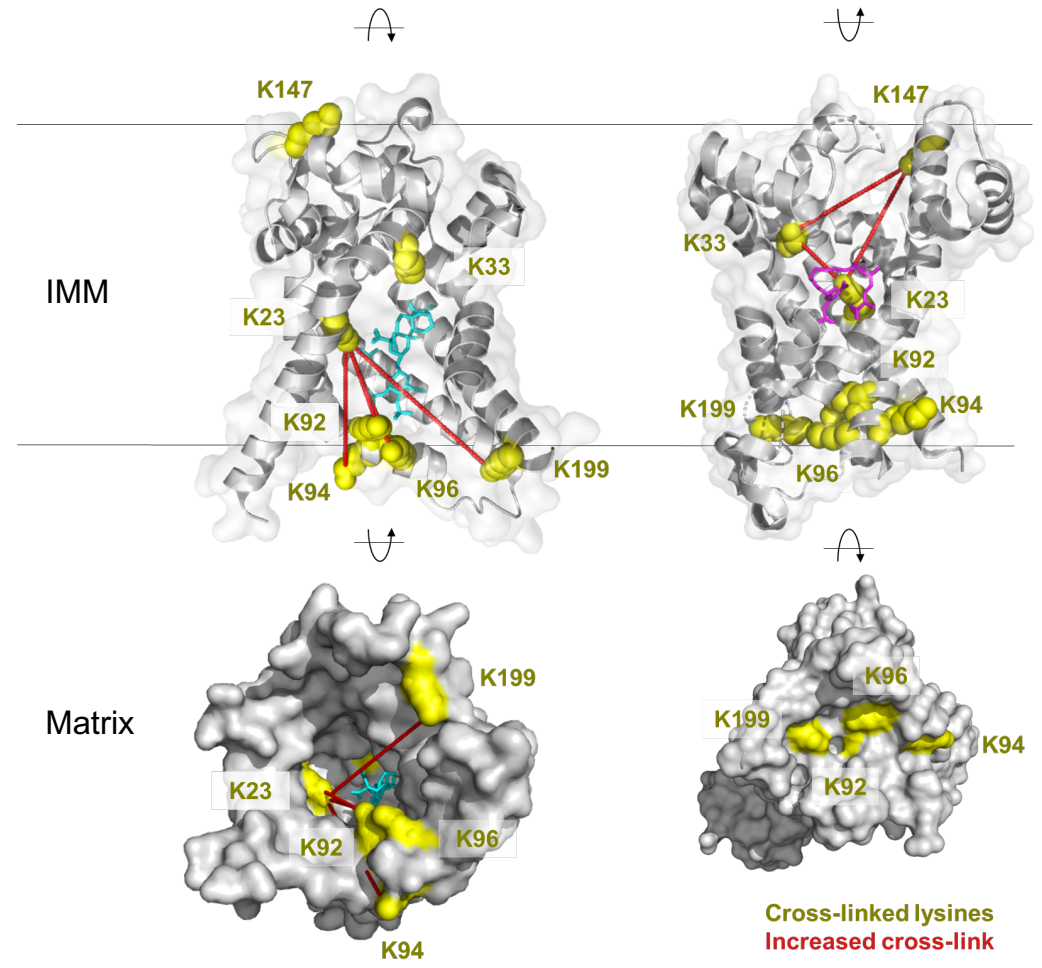


\section{Figure 4: Enrichment of an intermediate state of ADPIATP carrier detected in TAC}

(A) Schematic of ADT cycling between two dominant conformational states. The cytoplasmicfacing state (C-state) delivers ATP to the cytosol and binds ADP. Binding of ADP induces a conformational change to the matrix-facing state (M-state), which returns ADP to the mitochondria and binds a newly charged ATP.

(B) Table summarizing mean cross-linking ratio and DE level ratio for each cross-linked peptide pair detected in ADT1 exhibiting changes in TAC. Euclidean distance (EI) and Solvent Accessible Surface distance (Jwalk SASD) (Bullock et al., 2016) are listed for each crosslinked peptide pair when mapped onto both C-state and M-state of ADT1. Highlighted values satisfy iqPIR molecular distance constraint (EI<42A, Jwalk SASD $<51 \AA$ ).

(C) Cross-linked peptide pairs (yellow lysine side chains) mapped onto the C-state conformation of bovine ADT1 (PDB: 1OKC). In this conformation, K23, K92, K94, K96, and K199 are available and can satisfy cross-linking distance constraints. K147 and K33 are not accessible in the M-state. Carboxyatractyloside (CATR) stabilizes the C-state and is shown in cyan.

(D) Cross-linked peptide pairs (yellow lysine side chains) mapped onto the M-state of ADT1 (TtAac PDB: 6GCI) where residues K92, K94, K96 and K199 form the gate closed to the matrix, and are inaccessible for cross-linking.Three cross-links are possible and shown between K23-K33, K23-K147, and K33-K147. Bongkrekic acid (BKA) stabilizes the M-state and is shown in magenta. 


\section{Supplemental Figure 1}

A

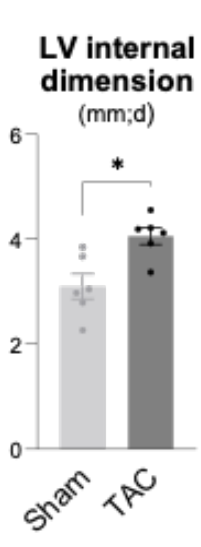

B

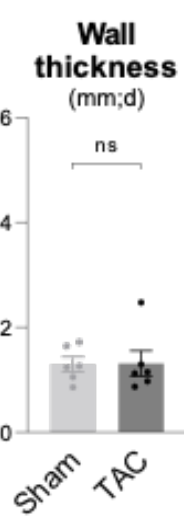

C

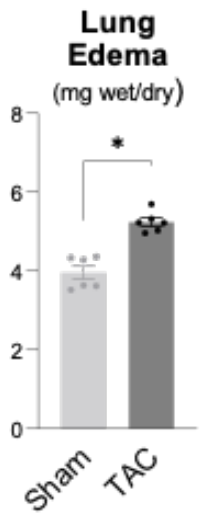

D

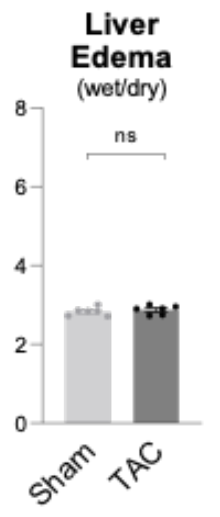

E

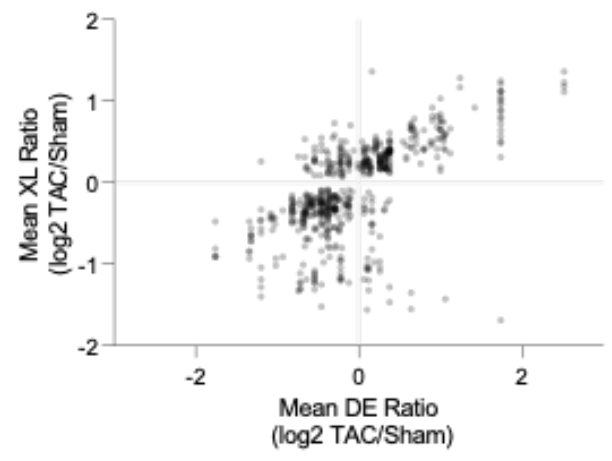

$\mathbf{F}$

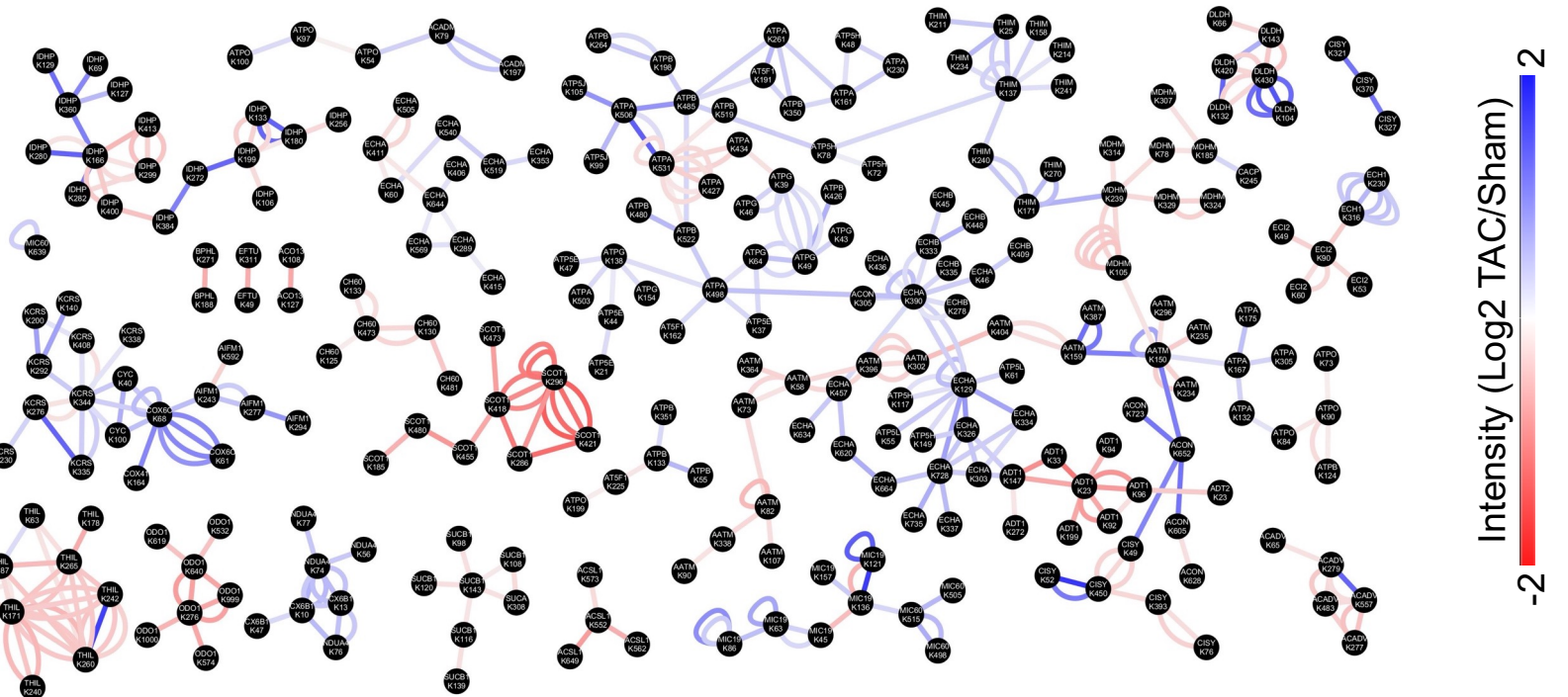

(2)

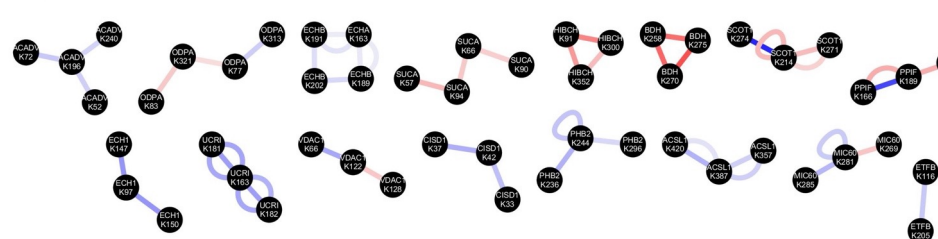

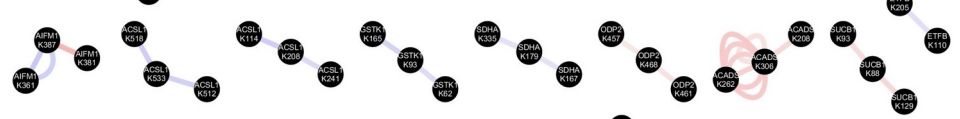

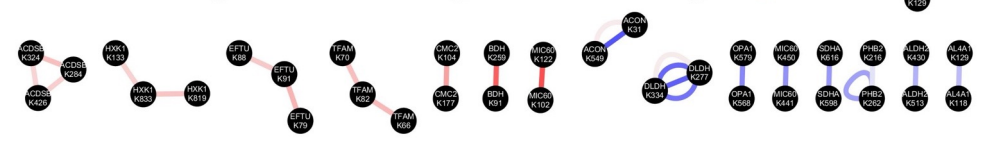

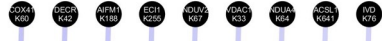

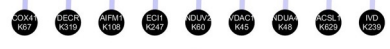
$\rightarrow \circ \rightarrow \infty$

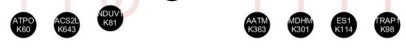

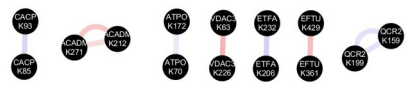
$\because \rightarrow \circ: \circ 0$

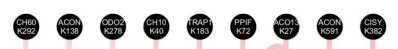

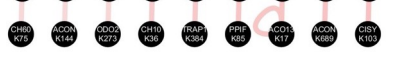




\section{Supplemental Figure 1}

(A-B) Left ventricle internal dimension and wall thickness in TAC and Sham groups determined by echocardiography four weeks post-surgery.

(C-D) Lung and liver edema (wet weight/dry weight in $\mathrm{mg}$ ) measured at tissue harvest.

(E) Quantitation of mean cross-link (XL) ratio vs mean dead-end (DE) ratio for each crosslinked peptide pair statistically changed in TAC in at least 4/6 biological pairs (Log2 TAC/Sham). Sum of Mean DE ratio for Protein A and Protein B is shown to account for crosslinks between two different proteins.

(F) Interaction network of lysine residues (black nodes) connected by observed cross-links (edges). Edges are colored according to increasing (red) and decreasing (blue) quantitation of statistically significant subset of cross-links (Log2 TAC/Sham) shown by color scale.

For (A-D), all data are $n=6, A V G+/-S E M,{ }^{*}$ denotes $p<0.05$ by Student's $t$ test. 


\section{Supplemental Figure 2}

A

\begin{tabular}{|c|c|c|c|c|c|c|c|c|c|c|}
\hline \multicolumn{3}{|c|}{ Cross-linked peptide pair } & \multicolumn{8}{|c|}{ Log2 ratio (TAC/Sham) } \\
\hline Protein & LysA & LysB & $\begin{array}{c}3702 \mathrm{RH} \\
\mathrm{XL}\end{array}$ & $\begin{array}{c}3707 R H \\
\text { XL }\end{array}$ & $\begin{array}{c}3713 \mathrm{RH} \\
\mathrm{XL}\end{array}$ & $\begin{array}{c}3705 S H \\
X L\end{array}$ & $\begin{array}{c}3712 \mathrm{SH} \\
\mathrm{XL}\end{array}$ & $\begin{array}{c}3752 \mathrm{SH} \\
\mathrm{xL}\end{array}$ & $\begin{array}{c}\text { Mean } \\
\text { XL }\end{array}$ & $\begin{array}{c}\text { Mean } \\
\text { DE }\end{array}$ \\
\hline SCOT1 & 296 & 421 & 1.36 & 1.44 & 1.17 & 1.26 & 1.23 & 1.04 & 1.25 & 0.87 \\
\hline SCOT1 & 286 & 418 & 1.08 & 1.46 & 0.98 & 1.04 & 0.87 & 0.87 & 1.02 & 0.87 \\
\hline SCOT1 & 296 & 418 & 0.76 & 1.52 & 0.95 & 0.88 & 1.03 & 1.07 & 0.99 & 0.87 \\
\hline SCOT1 & 286 & 421 & 1.31 & 1.6 & 1.09 & 0.89 & 1.26 & 1.01 & 1.12 & 0.87 \\
\hline SCOT1 & 296 & 296 & & 1.13 & 1.08 & 0.86 & 1.03 & & 1.05 & 0.87 \\
\hline SCOT1 & 214 & 274 & & -1.84 & & -1.12 & -1 & -1.57 & -1.69 & 0.87 \\
\hline THIL & 260 & 242 & & -1.42 & & -1.59 & -0.69 & -1.55 & -1.47 & 0.19 \\
\hline
\end{tabular}

B

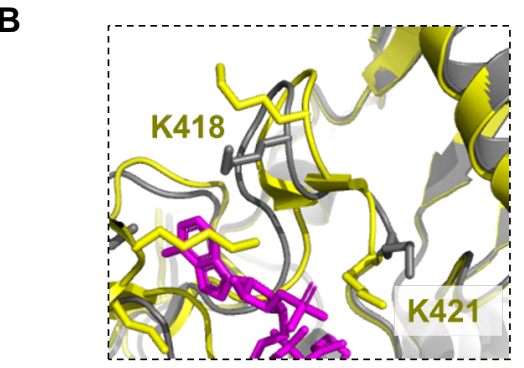

No substrate

CoA bound

CoA
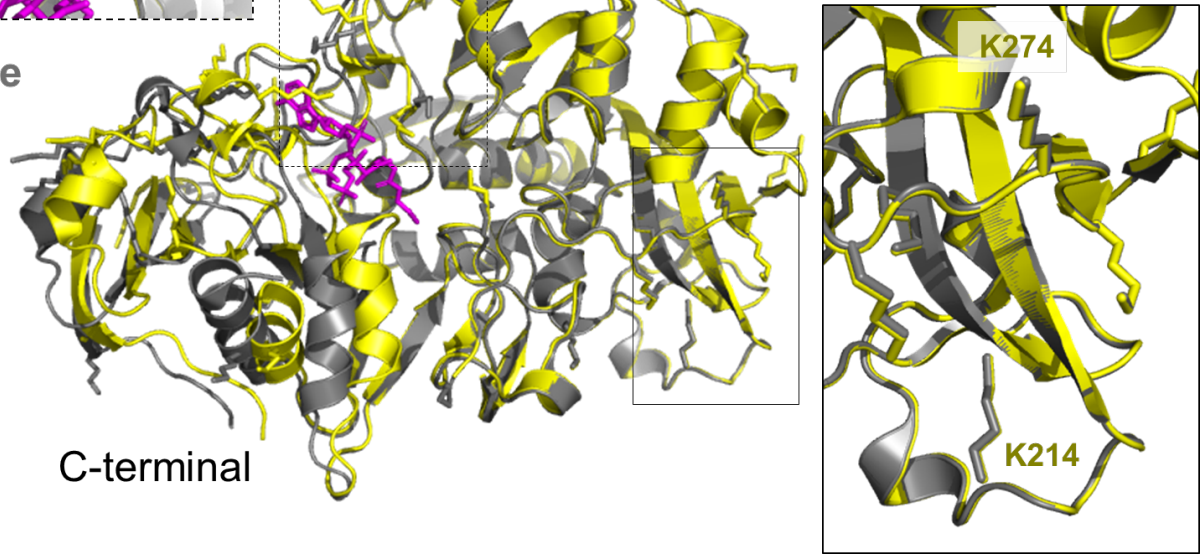

C

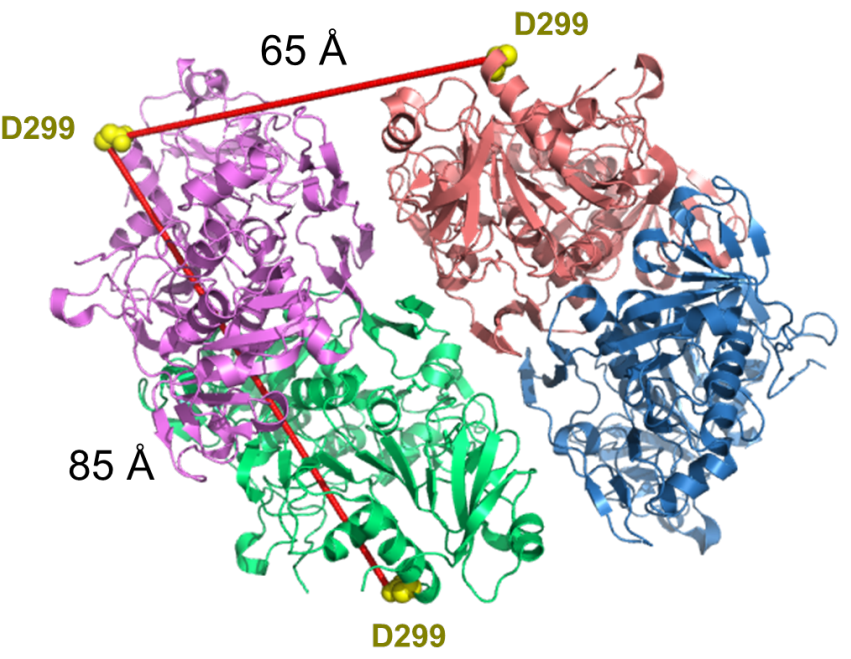




\section{Supplemental Figure 2}

(A) Table summarizing the mean cross-linking ratio and DE ratio for cross-linked peptide pairs including values obtained across biological replicates for ketone oxidation proteins.

(B) Alignment of apo (grey, PDB: $30 X 0$ chain A) and substrate-bound (yellow, PDB: $30 X 0$ chain E) monomers of porcine SCOT1. CoA is colored in magenta and bound to the active site. Lysine sidechains are shown in stick representation. Alignment depicts the structural differences between the dynamic C-terminal domain and the static $\mathrm{N}$-terminal domain during substrate-binding. Close up view specifies cross-linked lysines (K214, K274, K418, and K421).

(C) Human SCOT1 tetrameric structure (PDB: 3DLX) illustrating the observed K296-K296 homodimer cross-link that increases in TAC. Molecular interaction distance constraint is exceeded when mapped onto the tetrameric structure. Each monomer is depicted in a different color. K296 is not present in electron density of solved structures, closest resolved residue present in each monomer is shown (D299). 


\section{Supplemental Figure 3}

A
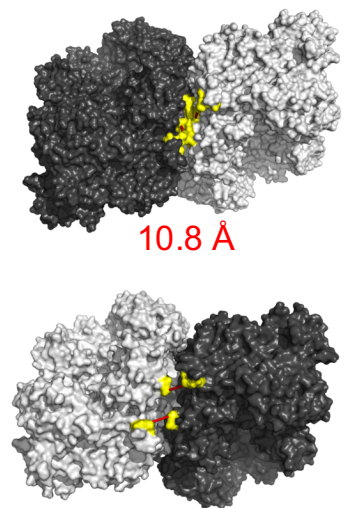

$12.8 \AA$
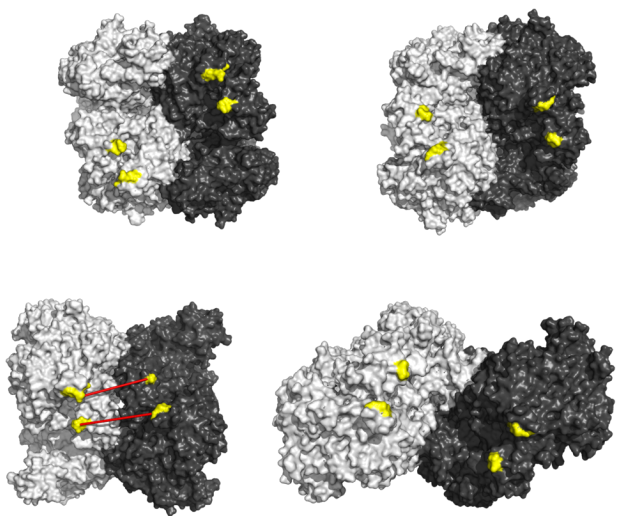

$40.9 \AA$
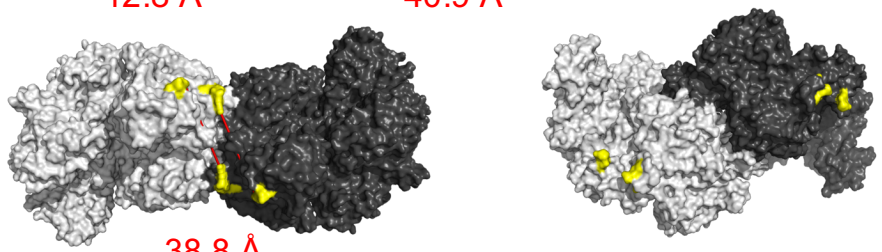

$38.8 \AA$

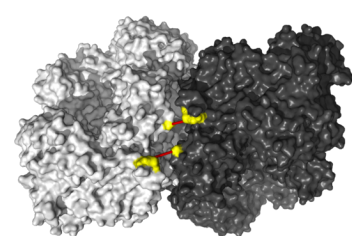

$12.5 \AA$

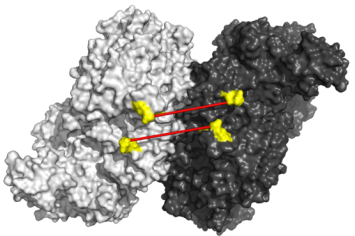

$32.6 \AA$

B
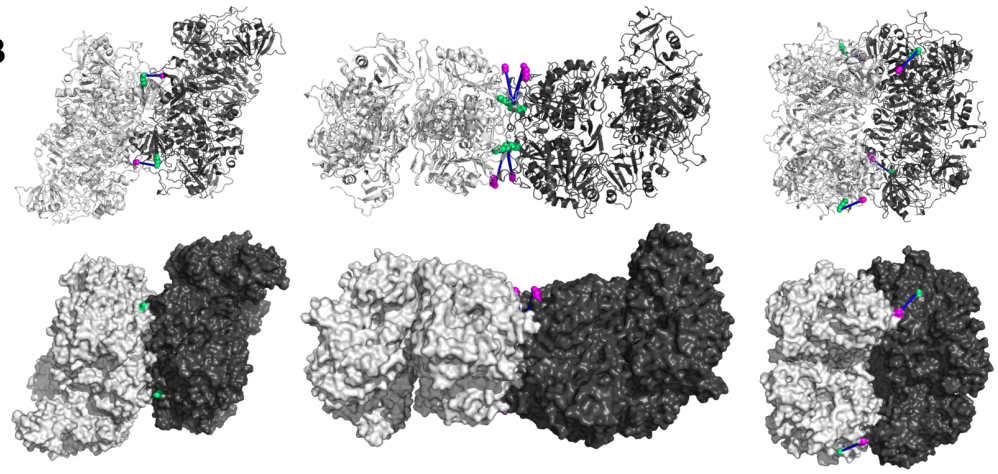

C

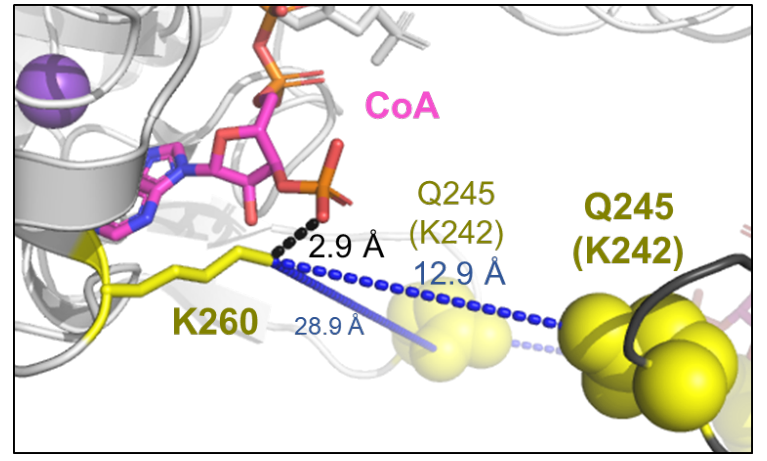




\section{Supplemental Figure 3}

(A) Top 10 solutions from molecular docking of two human SCOT1 tetramers (PDB: 3DLX) using Symmdock (Schneidman-Duhovny et al., 2005). Approximate location of K296-K296 was examined in each solution. Due to the missing density around K296, residues $284-302$ were highlighted (yellow). In 6 of the top 10 solutions, proximity between two tetramers is consistent with molecular distance constraint of cross-linker (listed below structure).

(B) Three (of top ten) docking solutions where residues K214 or K274 are buried at the interface between two SCOT1 tetramers (PDB: 3DLX). Decreasing cross-link between K214 (green) and K274 (magenta) is shown as a blue edge.

(C) Human THIL (PDB: 2IBW) tetrameric structure highlighting the salt bridge formed between K260 (yellow stick representation) and 3' phosphate moiety of CoA (2.9 $\AA)$, which may make K260 inaccessible for cross-linking with an adjacent K242/Q245 (yellow sphere) (intra-link distance $28.9 \AA$ ) or K242/KQ245 on the opposing monomer (inter-link distance $12.9 \AA$ ). 


\section{Supplemental Figure 4}

A

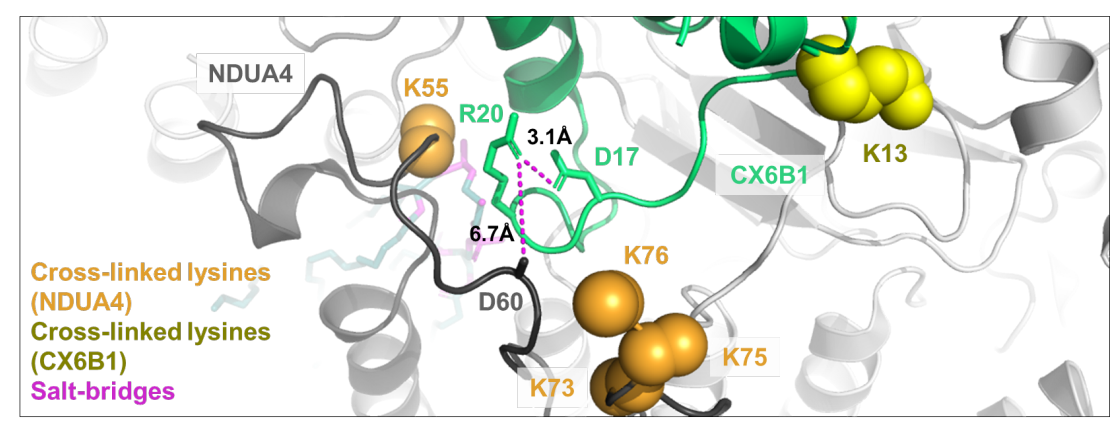

B

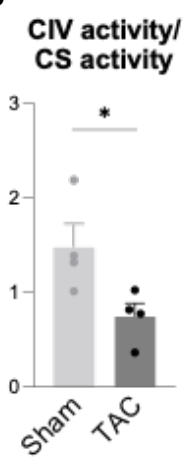

C

\begin{tabular}{|c|c|c|c|c|c|c|c|c|c|c|c|c|c|c|}
\hline \multicolumn{3}{|c|}{ Cross-linked peptide pair } & \multicolumn{8}{|c|}{ Log2 ratio (TAC/Sham) } & \multicolumn{2}{|c|}{ C-state } & \multicolumn{2}{|c|}{ M-state } \\
\hline Protein & LysA & LysB & $\begin{array}{c}3702 R H \\
X L\end{array}$ & $\begin{array}{c}\text { 3707RH } \\
X L\end{array}$ & $\begin{array}{c}3713 R H \\
X L\end{array}$ & $\begin{array}{c}3705 S H \\
X L\end{array}$ & $\begin{array}{c}3712 \mathrm{SH} \\
\mathrm{XL}\end{array}$ & $\begin{array}{c}3752 \mathrm{SH} \\
\mathrm{XL}\end{array}$ & $\begin{array}{c}\text { Mean } \\
\mathrm{XL}\end{array}$ & $\begin{array}{c}\text { Mean } \\
\mathrm{DE}\end{array}$ & \begin{tabular}{|c|}
$\begin{array}{c}\text { Euclidean } \\
\text { distance }\end{array}$ \\
\end{tabular} & $\begin{array}{l}\text { Jwalk } \\
\text { SASD }\end{array}$ & $\begin{array}{c}\text { Euclidean } \\
\text { distance }\end{array}$ & $\begin{array}{l}\text { Jwalk } \\
\text { SASD }\end{array}$ \\
\hline ADT1 & 92 & 23 & 0.82 & 0.34 & 0.91 & 0.48 & 1.13 & 0.65 & 0.74 & 0.5 & 22.6 & 23.78 & 20.08 & 99 \\
\hline ADT1 & 94 & 23 & 0.96 & 0.4 & 0.3 & 0.21 & 1.05 & 0.35 & 0.55 & 0.5 & 28.13 & 31.94 & 24.1 & 61.38 \\
\hline ADT1 & 96 & 23 & 0.71 & 0.35 & 1.06 & 0.49 & 1.15 & 0.69 & 0.79 & 0.5 & 27.32 & 29.61 & 25.47 & 99 \\
\hline ADT1 & 199 & 23 & 1.14 & 0.39 & 1.05 & 0.41 & 1.07 & 0.92 & 0.65 & 0.5 & 27.96 & 32.09 & 22.62 & 99 \\
\hline ADT1 & 33 & 23 & & 0.82 & 1.04 & 1.09 & 1.39 & & 1.03 & 0.5 & 13.52 & 16.64 & 14.41 & 17.78 \\
\hline ADT1 & 147 & 23 & & 0.86 & 0.54 & 0.73 & 1 & & 0.65 & 0.5 & 23.53 & 66.86 & 27.25 & 30.92 \\
\hline ADT1 & 147 & 33 & 1.14 & 0.39 & 1.05 & 0.41 & 1.07 & 0.92 & 0.65 & 0.5 & 19.12 & 99 & 24.51 & 31.65 \\
\hline
\end{tabular}

D

\begin{tabular}{|c|c|c|c|c|c|c|c|c|c|c|c|c|}
\hline \multicolumn{4}{|c|}{ Cross-linked peptide pair } & \multicolumn{9}{|c|}{ Log2 ratio (TAC/Sham) } \\
\hline & & & & $3702 \mathrm{RH}$ & $3707 \mathrm{RH}$ & $3713 R H$ & $3705 \mathrm{SH}$ & $3712 \mathrm{SH}$ & $3752 S$ & Mean & Mean DE & Mean DE \\
\hline ProteinA & ProteinB & LysA & LysB & $\mathrm{XL}$ & $\mathrm{xL}$ & $\mathrm{XL}$ & $\mathrm{XL}$ & $\mathrm{xL}$ & $\mathrm{HXL}$ & $\mathrm{XL}$ & A & B \\
\hline ADT1 & ADT2 & 96 & 23 & 1.08 & 0.22 & 0.3 & 0.65 & 1.16 & 0.71 & 0.36 & 0.5 & 0.62 \\
\hline ADT2 & ADT2 & 96 & 23 & & 0.81 & 1.51 & 0.82 & 1.78 & & 1.28 & 0.62 & 0.62 \\
\hline ADT2 & ADT2 & 199 & 23 & & 1.16 & 1.25 & & 2.64 & 0.89 & 1.17 & 0.62 & 0.62 \\
\hline
\end{tabular}

E

\section{TtAac M-state}

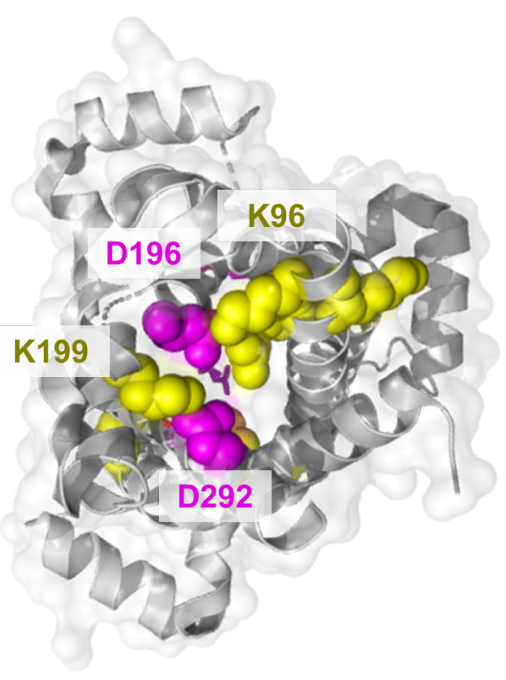

$\mathbf{F}$

Bovine C-state 


\section{Supplemental Figure 4}

(A) Structural insight into CX6B1 R20 forming salt-bridges at NDUA4-CX6B1 interface. R20 sidechain (green) forms a salt-bridge with CX6B1 D16 (green) and NDUA4 D60 (black), which pinpoints an interface necessary for stability of CIV. Side-chains of R20, D16, and NDUA4 D60 (partially resolved) are depicted in stick representation. Cross-linked lysine sidechains are shown as yellow (CX6B1) or orange (NDUA4) spheres.

(B) Cytochrome C oxidase enzymatic activity assay in tissue homogenates from TAC and Sham hearts, normalized to Citrate Synthase activity. N=4, AVG+/-SEM, *denotes $p<0.05$ by Student's t test.

(C) Table summarizing the mean cross-linking ratio and DE ratio for cross-linked peptide pairs including values obtained across biological replicates for ADT1.

(D) Table summarizing the mean cross-linking ratio and DE ratio for cross-linked peptide pairs including values obtained across biological replicates for cross-links in ADT isoforms.

(E) Cross-linked peptide pairs (yellow lysine side chains) mapped onto the M-state conformation of ADT1 (TtAac PDB: 6GCI). Salt-bridges between K96-D196 and K199-D292 contribute to the gating mechanism which closes the M-state to the IMS (Ruprecht et al., 2019), and would make lysines unavailable for cross-linking. Aspartic acid sidechains are shown in magenta.

(F) Cross-linked peptide pairs (yellow lysine side chains) mapped onto the C-state conformation of bovine ADT1 (PDB: 1OKC). K33 and D231 are known to form a salt-bridge which stabilizes the C-state (Pebay-Peyroula et al., 2003) and would make K33 unavailable for cross-linking. Aspartic acid sidechains are shown in magenta. 


\section{Supplemental Video 1}

This video can be viewed by anyone with the following link:

https://drive.google.com/file/d/1G8NtkRJylq1xKNzMa4ovpwFlupzenzht/view?usp=sharing

\section{References}

1. Abdulhag, U.N., Soiferman, D., Schueler-Furman, O., Miller, C., Shaag, A., Elpeleg, O., Edvardson, S., and Saada, A. (2015). Mitochondrial complex IV deficiency, caused by mutated COX6B1, is associated with encephalomyopathy, hydrocephalus and cardiomyopathy. European Journal of Human Genetics 23, 159-164. 10.1038/ejhg.2014.85.

2. Aubert, G., Martin Ola, J., Horton Julie, L., Lai, L., Vega Rick, B., Leone Teresa, C., Koves, T., Gardell Stephen, J., Krüger, M., Hoppel Charles, L., et al. (2016). The Failing Heart Relies on Ketone Bodies as a Fuel. Circulation 133, 698-705. 10.1161/CIRCULATIONAHA.115.017355.

3. Balsa, E., Marco, R., Perales-Clemente, E., Szklarczyk, R., Calvo, E., Landázuri, Manuel O., and Enríquez, José A. (2012). NDUFA4 Is a Subunit of Complex IV of the Mammalian Electron Transport Chain. Cell Metabolism 16, 378-386. 10.1016/j.cmet.2012.07.015.

4. Barth, E., Stämmler, G., Speiser, B., and Schaper, J. (1992). Ultrastructural quantitation of mitochondria and myofilaments in cardiac muscle from 10 different animal species including man. Journal of Molecular and Cellular Cardiology 24, 669-681. https://doi.org/10.1016/0022-2828(92)93381-S.

5. Bedi, K.C., Snyder, N.W., Brandimarto, J., Aziz, M., Mesaros, C., Worth, A.J., Wang, L.L., Javaheri, A., Blair, I.A., Margulies, K.B., and Rame, J.E. (2016). Evidence for Intramyocardial Disruption of Lipid Metabolism and Increased Myocardial Ketone Utilization in Advanced Human Heart Failure. Circulation 133, 706-716. 10.1161/CIRCULATIONAHA.115.017545.

6. Bertholet, A.M., Chouchani, E.T., Kazak, L., Angelin, A., Fedorenko, A., Long, J.Z., Vidoni, S., Garrity, R., Cho, J., Terada, N., et al. (2019). H+ transport is an integral function of the mitochondrial ADP/ATP carrier. Nature 571, 515-520. 10.1038/s41586019-1400-3.

7. Bridges, H.R., Fedor, J.G., Blaza, J.N., Di Luca, A., Jussupow, A., Jarman, O.D., Wright, J.J., Agip, A.-N.A., Gamiz-Hernandez, A.P., Roessler, M.M., et al. (2020). Structure of inhibitor-bound mammalian complex I. Nature Communications 11, 5261. 10.1038/s41467-020-18950-3.

8. Broom, B.M., Ryan, M.C., Brown, R.E., Ikeda, F., Stucky, M., Kane, D.W., Melott, J., Wakefield, C., Casasent, T.D., Akbani, R., and Weinstein, J.N. (2017). A Galaxy Implementation of Next-Generation Clustered Heatmaps for Interactive Exploration of Molecular Profiling Data. Cancer Research 77, e23. 10.1158/0008-5472.CAN-17-0318. 
9. Bround, M.J., Bers, D.M., and Molkentin, J.D. (2020). A 20/20 view of ANT function in mitochondrial biology and necrotic cell death. Journal of Molecular and Cellular Cardiology 144, A3-A13. 10.1016/j.yjmcc.2020.05.012.

10.Brown, D.A., Perry, J.B., Allen, M.E., Sabbah, H.N., Stauffer, B.L., Shaikh, S.R., Cleland, J.G.F., Colucci, W.S., Butler, J., Voors, A.A., et al. (2017). Mitochondrial function as a therapeutic target in heart failure. Nature Reviews Cardiology 14, 238-250. 10.1038/nrcardio.2016.203.

11. Bullock, J.M.A., Schwab, J., Thalassinos, K., and Topf, M. (2016). The Importance of Non-accessible Crosslinks and Solvent Accessible Surface Distance in Modeling Proteins with Restraints From Crosslinking Mass Spectrometry *<sup $></$ sup $>$. Molecular \& Cellular Proteomics 15, 2491-2500. 10.1074/mcp.M116.058560.

12. Calvo, S.E., Clauser, K.R., and Mootha, V.K. (2015). MitoCarta2.0: an updated inventory of mammalian mitochondrial proteins. Nucleic Acids Research 44, D1251D1257. 10.1093/nar/gkv1003.

13. Chavez, J.D., Cilia, M., Weisbrod, C.R., Ju, H.-J., Eng, J.K., Gray, S.M., and Bruce, J.E. (2012). Cross-linking measurements of the Potato leafroll virus reveal protein interaction topologies required for virion stability, aphid transmission, and virus-plant interactions. Journal of proteome research 11, 2968-2981. 10.1021/pr300041t.

14. Chavez, J.D., Keller, A., Mohr, J.P., and Bruce, J.E. (2020a). Isobaric Quantitative Protein Interaction Reporter Technology for Comparative Interactome Studies. Analytical Chemistry 92, 14094-14102. 10.1021/acs.analchem.0c03128.

15. Chavez, J.D., Keller, A., Zhou, B., Tian, R., and Bruce, J.E. (2019a). Cellular Interactome Dynamics during Paclitaxel Treatment. Cell Reports 29, 2371-2383.e2375. 10.1016/j.celrep.2019.10.063.

16. Chavez, J.D., Lee, C.F., Caudal, A., Keller, A., Tian, R., and Bruce, J.E. (2018). Chemical Crosslinking Mass Spectrometry Analysis of Protein Conformations and Supercomplexes in Heart Tissue. Cell Systems 6, 136-141.e135. 10.1016/j.cels.2017.10.017.

17. Chavez, J.D., Mohr, J.P., Mathay, M., Zhong, X., Keller, A., and Bruce, J.E. (2019b). Systems structural biology measurements by in vivo cross-linking with mass spectrometry. Nature Protocols 14, 2318-2343. 10.1038/s41596-019-0181-3.

18. Chavez, Juan D., Schweppe, Devin K., Eng, Jimmy K., and Bruce, James E. (2016). In Vivo Conformational Dynamics of Hsp90 and Its Interactors. Cell Chemical Biology 23, 716-726. https://doi.org/10.1016/j.chembiol.2016.05.012.

19. Chavez, J.D., Schweppe, D.K., Eng, J.K., Zheng, C., Taipale, A., Zhang, Y., Takara, K., and Bruce, J.E. (2015). Quantitative interactome analysis reveals a chemoresistant edgotype. 6, 7928. 10.1038/ncomms8928

https://www.nature.com/articles/ncomms8928\#supplementary-information.

20. Chavez, J.D., Tang, X., Campbell, M.D., Reyes, G., Kramer, P.A., Stuppard, R., Keller, A., Zhang, H., Rabinovitch, P.S., Marcinek, D.J., and Bruce, J.E. (2020b). Mitochondrial protein interaction landscape of SS-31. Proceedings of the National Academy of Sciences 117, 15363. 10.1073/pnas.2002250117.

21. Chavez, J.D., Weisbrod, C.R., Zheng, C., Eng, J.K., and Bruce, J.E. (2013). Protein Interactions, Post-translational Modifications and Topologies in Human Cells * <sup $>$ \&\#x9;\&\#x9;</sup>. Molecular \& Cellular Proteomics 12, 1451-1467.

10.1074/mcp.M112.024497. 
22. Coker, S.-F., Lloyd, A.J., Mitchell, E., Lewis, G.R., Coker, A.R., and Shoolingin-Jordan, P.M. (2010). The high-resolution structure of pig heart succinyl-CoA:3-oxoacid coenzyme A transferase. Acta Crystallographica Section D 66, 797-805. doi:10.1107/S0907444910018366.

23. Dittenhafer-Reed, Kristin E., Richards, Alicia L., Fan, J., Smallegan, Michael J., Fotuhi Siahpirani, A., Kemmerer, Zachary A., Prolla, Tomas A., Roy, S., Coon, Joshua J., and Denu, John M. (2015). SIRT3 Mediates Multi-Tissue Coupling for Metabolic Fuel Switching. Cell Metabolism 21, 637-646. 10.1016/j.cmet.2015.03.007.

24. Fraser, M.E., Hayakawa, K., and Brown, W.D. (2010). Catalytic Role of the Conformational Change in Succinyl-CoA:3-Oxoacid CoA Transferase on Binding CoA. Biochemistry 49, 10319-10328. 10.1021/bi100659s.

25. Gu, J., Zhang, L., Zong, S., Guo, R., Liu, T., Yi, J., Wang, P., Zhuo, W., and Yang, M. (2019). Cryo-EM structure of the mammalian ATP synthase tetramer bound with inhibitory protein IF1. Science 364, 1068. 10.1126/science.aaw4852.

26. Haapalainen, A.M., Meriläinen, G., Pirilä, P.L., Kondo, N., Fukao, T., and Wierenga, R.K. (2007). Crystallographic and Kinetic Studies of Human Mitochondrial AcetoacetylCoA Thiolase: The Importance of Potassium and Chloride lons for Its Structure and Function. Biochemistry 46, 4305-4321. 10.1021/bi6026192.

27. Karch, J., Bround, M.J., Khalil, H., Sargent, M.A., Latchman, N., Terada, N., Peixoto, P.M., and Molkentin, J.D. (2019). Inhibition of mitochondrial permeability transition by deletion of the ANT family and CypD. Science Advances 5, eaaw4597. 10.1126/sciadv.aaw4597.

28. Keller, A., Chavez, J.D., and Bruce, J.E. (2018). Increased sensitivity with automated validation of XL-MS cleavable peptide crosslinks. Bioinformatics 35, 895-897. 10.1093/bioinformatics/bty720.

29. Keller, A., Eng, J., Zhang, N., Li, X.-j., and Aebersold, R. (2005). A uniform proteomics MS/MS analysis platform utilizing open XML file formats. Molecular Systems Biology 1, 2005.0017. https://doi.org/10.1038/msb4100024.

30. Keller, A., Nesvizhskii, A.I., Kolker, E., and Aebersold, R. (2002). Empirical Statistical Model To Estimate the Accuracy of Peptide Identifications Made by MS/MS and Database Search. Analytical Chemistry 74, 5383-5392. 10.1021/ac025747h.

31. Kokoszka, J.E., Waymire, K.G., Levy, S.E., Sligh, J.E., Cai, J., Jones, D.P., MacGregor, G.R., and Wallace, D.C. (2004). The ADP/ATP translocator is not essential for the mitochondrial permeability transition pore. Nature 427, 461-465. 10.1038/nature02229.

32. Kolwicz, S.C., Airhart, S., and Tian, R. (2016). Ketones Step to the Plate. Circulation 133, 689-691. doi:10.1161/CIRCULATIONAHA.116.021230.

33. Massa, V., Fernandez-Vizarra, E., Alshahwan, S., Bakhsh, E., Goffrini, P., Ferrero, I., Mereghetti, P., D'Adamo, P., Gasparini, P., and Zeviani, M. (2008). Severe infantile encephalomyopathy caused by a mutation in COX6B1, a nucleus-encoded subunit of cytochrome c oxidase. American journal of human genetics 82, 1281-1289.

10.1016/j.ajhg.2008.05.002.

34. Mohr, J.P., Perumalla, P., Chavez, J.D., Eng, J.K., and Bruce, J.E. (2018). Mango: A General Tool for Collision Induced Dissociation-Cleavable Cross-Linked Peptide Identification. Analytical Chemistry 90, 6028-6034. 10.1021/acs.analchem.7b04991.

35. Murashige, D., Jang, C., Neinast, M., Edwards, J.J., Cowan, A., Hyman, M.C., Rabinowitz, J.D., Frankel, D.S., and Arany, Z. (2020). Comprehensive quantification of 
fuel use by the failing and nonfailing human heart. Science 370, 364 . 10.1126/science.abc8861.

36. Ong, S.-E., Blagoev, B., Kratchmarova, I., Kristensen, D.B., Steen, H., Pandey, A., and Mann, M. (2002). Stable Isotope Labeling by Amino Acids in Cell Culture, SILAC, as a Simple and Accurate Approach to Expression Proteomics*. Molecular \& Cellular Proteomics 1, 376-386. https://doi.org/10.1074/mcp.M200025-MCP200.

37.Pagliarini, D.J., Calvo, S.E., Chang, B., Sheth, S.A., Vafai, S.B., Ong, S.-E., Walford, G.A., Sugiana, C., Boneh, A., Chen, W.K., et al. (2008). A Mitochondrial Protein Compendium Elucidates Complex I Disease Biology. Cell 134, 112-123. 10.1016/j.cell.2008.06.016.

38. Pebay-Peyroula, E., Dahout-Gonzalez, C., Kahn, R., Trézéguet, V., Lauquin, G.J.M., and Brandolin, G. (2003). Structure of mitochondrial ADP/ATP carrier in complex with carboxyatractyloside. Nature 426, 39-44. 10.1038/nature02056.

39. Perez-Riverol, Y., Csordas, A., Bai, J., Bernal-Llinares, M., Hewapathirana, S., Kundu, D.J., Inuganti, A., Griss, J., Mayer, G., Eisenacher, M., et al. (2018). The PRIDE database and related tools and resources in 2019: improving support for quantification data. Nucleic Acids Research 47, D442-D450. 10.1093/nar/gky1106.

40. Qi, L., Wang, Q., Guan, Z., Wu, Y., Shen, C., Hong, S., Cao, J., Zhang, X., Yan, C., and Yin, P. (2021). Cryo-EM structure of the human mitochondrial translocase TIM22 complex. Cell Research 31, 369-372. 10.1038/s41422-020-00400-w.

41. Ramzan, R., Rhiel, A., Weber, P., Kadenbach, B., and Vogt, S. (2019). Reversible dimerization of cytochrome $\mathrm{c}$ oxidase regulates mitochondrial respiration. Mitochondrion 49, 149-155. https://doi.org/10.1016/j.mito.2019.08.002.

42. Rath, S., Sharma, R., Gupta, R., Ast, T., Chan, C., Durham, T.J., Goodman, R.P., Grabarek, Z., Haas, M.E., Hung, W.H.W., et al. (2020). MitoCarta3.0: an updated mitochondrial proteome now with sub-organelle localization and pathway annotations. Nucleic Acids Research 49, D1541-D1547. 10.1093/nar/gkaa1011.

43. Ritterhoff, J., Young, S., Villet, O., Shao, D., Neto, F.C., Bettcher Lisa, F., Hsu Yun-Wei, A., Kolwicz Stephen, C., Raftery, D., and Tian, R. (2020). Metabolic Remodeling Promotes Cardiac Hypertrophy by Directing Glucose to Aspartate Biosynthesis. Circulation Research 126, 182-196. 10.1161/CIRCRESAHA.119.315483.

44. Rogers, G.W., Brand, M.D., Petrosyan, S., Ashok, D., Elorza, A.A., Ferrick, D.A., and Murphy, A.N. (2011). High Throughput Microplate Respiratory Measurements Using Minimal Quantities Of Isolated Mitochondria. PLOS ONE 6, e21746. 10.1371/journal.pone.0021746.

45. Rosca, M.G., Vazquez, E.J., Kerner, J., Parland, W., Chandler, M.P., Stanley, W., Sabbah, H.N., and Hoppel, C.L. (2008). Cardiac mitochondria in heart failure: decrease in respirasomes and oxidative phosphorylation. Cardiovascular Research 80, 30-39. 10.1093/cvr/cvn184.

46. Ross, P.L., Huang Yn Fau - Marchese, J.N., Marchese Jn Fau - Williamson, B., Williamson B Fau - Parker, K., Parker K Fau - Hattan, S., Hattan S Fau - Khainovski, N., Khainovski N Fau - Pillai, S., Pillai S Fau - Dey, S., Dey S Fau - Daniels, S., Daniels S Fau - Purkayastha, S., et al. Multiplexed protein quantitation in Saccharomyces cerevisiae using amine-reactive isobaric tagging reagents. 
47. Ruprecht, J.J., King, M.S., Zögg, T., Aleksandrova, A.A., Pardon, E., Crichton, P.G., Steyaert, J., and Kunji, E.R.S. (2019). The Molecular Mechanism of Transport by the Mitochondrial ADP/ATP Carrier. Cell 176, 435-447.e415. 10.1016/j.cell.2018.11.025.

48. Schneidman-Duhovny, D., Inbar, Y., Nussinov, R., and Wolfson, H.J. (2005). PatchDock and SymmDock: servers for rigid and symmetric docking. Nucleic Acids Research 33, W363-W367. 10.1093/nar/gki481.

49. Schweppe, D.K., Chavez, J.D., Lee, C.F., Caudal, A., Kruse, S.E., Stuppard, R., Marcinek, D.J., Shadel, G.S., Tian, R., and Bruce, J.E. (2017). Mitochondrial protein interactome elucidated by chemical cross-linking mass spectrometry. Proceedings of the National Academy of Sciences 114, 1732-1737. 10.1073/pnas.1617220114.

50. Schweppe, D.K., Zheng, C., Chavez, J.D., Navare, A.T., Wu, X., Eng, J.K., and Bruce, J.E. (2016). XLinkDB 2.0: integrated, large-scale structural analysis of protein crosslinking data. Bioinformatics 32, 2716-2718. 10.1093/bioinformatics/btw232.

51. Shafqat, N., Kavanagh, K.L., Sass, J.O., Christensen, E., Fukao, T., Lee, W.H., Oppermann, U., and Yue, W.W. (2013). A structural mapping of mutations causing succinyl-CoA:3-ketoacid CoA transferase (SCOT) deficiency. Journal of inherited metabolic disease 36, 983-987. 10.1007/s10545-013-9589-z.

52. Sinz, A. (2018). Crosslinking Mass Spectrometry Goes <em $>$ In-Tissue </em>. Cell Systems 6, 10-12. 10.1016/j.cels.2018.01.005.

53. Spikes, T.E., Montgomery, M.G., and Walker, J.E. (2020). Structure of the dimeric ATP synthase from bovine mitochondria. Proceedings of the National Academy of Sciences 117, 23519. 10.1073/pnas.2013998117.

54. Spinazzi, M., Casarin, A., Pertegato, V., Salviati, L., and Angelini, C. (2012). Assessment of mitochondrial respiratory chain enzymatic activities on tissues and cultured cells. Nature Protocols 7, 1235-1246. 10.1038/nprot.2012.058.

55. Still, A.J., Floyd, B.J., Hebert, A.S., Bingman, C.A., Carson, J.J., Gunderson, D.R., Dolan, B.K., Grimsrud, P.A., Dittenhafer-Reed, K.E., Stapleton, D.S., et al. (2013). Quantification of Mitochondrial Acetylation Dynamics Highlights Prominent Sites of Metabolic Regulation *<sup> \&\#x9;\&\#x9;</sup>. Journal of Biological Chemistry 288, 26209-26219. 10.1074/jbc.M113.483396.

56. Tammam, S.D., Rochet, J.-C., and Fraser, M.E. (2007). Identification of the Cysteine Residue Exposed by the Conformational Change in Pig Heart Succinyl-CoA:3-Ketoacid Coenzyme A Transferase on Binding Coenzyme A. Biochemistry 46, 10852-10863. 10.1021/bi700828h.

57.Tang, X., Munske, G.R., Siems, W.F., and Bruce, J.E. (2005). Mass Spectrometry Identifiable Cross-Linking Strategy for Studying Protein-Protein Interactions. Analytical Chemistry 77, 311-318. 10.1021/ac0488762.

58. Tarnavski, O., McMullen, J.R., Schinke, M., Nie, Q., Kong, S., and Izumo, S. (2004). Mouse cardiac surgery: comprehensive techniques for the generation of mouse models of human diseases and their application for genomic studies. Physiological Genomics 16, 349-360. 10.1152/physiolgenomics.00041.2003.

59. Thompson, A., Schäfer, J., Kuhn, K., Kienle, S., Schwarz, J., Schmidt, G., Neumann, T., and Hamon, C. (2003). Tandem Mass Tags: A Novel Quantification Strategy for Comparative Analysis of Complex Protein Mixtures by MS/MS. Analytical Chemistry 75, 1895-1904. 10.1021/ac0262560. 
60. Tucker, K., and Park, E. (2019). Cryo-EM structure of the mitochondrial protein-import channel TOM complex at near-atomic resolution. Nature Structural \& Molecular Biology 26, 1158-1166. 10.1038/s41594-019-0339-2.

61. Tyanova, S., Temu, T., Sinitcyn, P., Carlson, A., Hein, M.Y., Geiger, T., Mann, M., and Cox, J. (2016). The Perseus computational platform for comprehensive analysis of (prote)omics data. Nature Methods 13, 731-740. 10.1038/nmeth.3901.

62. Weisbrod, C.R., Chavez, J.D., Eng, J.K., Yang, L., Zheng, C., and Bruce, J.E. (2013). In Vivo Protein Interaction Network Identified with a Novel Real-Time Cross-Linked Peptide Identification Strategy. Journal of Proteome Research 12, 1569-1579. 10.1021/pr3011638.

63.Zong, S., Wu, M., Gu, J., Liu, T., Guo, R., and Yang, M. (2018). Structure of the intact 14-subunit human cytochrome c oxidase. Cell Research 28, 1026-1034.

10.1038/s41422-018-0071-1.

\section{STAR METHODS}

\section{KEY RESOURCES TABLE}

\begin{tabular}{|c|c|c|}
\hline REAGENT or RESOURCE & SOURCE & IDENTIFIER \\
\hline \multicolumn{3}{|c|}{ Chemicals, Peptides, and Recombinant proteins } \\
\hline iqPIR cross-linker & AnaSpec, Chavez et al 2020 & $\begin{array}{l}\text { Peptide sequence: Gly-GlyLys(biotin)- } \\
\text { Lys-Pro }_{2}-\mathrm{Asp}_{2} \text {-succinate } \\
\text {-N-N- } \\
\text { hydroxyphthalimide }(\mathrm{NHP})_{2}\end{array}$ \\
\hline \multicolumn{3}{|l|}{ Deposited Data } \\
\hline LC-MS data deposited into PRIDE & https://www.ebi.ac.uk/pride/archive/ & Accession No: PXD027757 \\
\hline Cross-link data deposited into XLinkDB & http://xlinkdb.gs.washington.edu/xlinkdb/index.php & $\begin{array}{l}\text { Network Name: } \\
\text { CaudalCellCommunity2021_Bruce }\end{array}$ \\
\hline \multicolumn{3}{|c|}{\begin{tabular}{l|l} 
Experimental Models: Organisms/Strains & \\
\end{tabular}} \\
\hline Mouse: C57BL6/NCrl & Charles River Laboratories & IMSR_CRL:27 \\
\hline \multicolumn{3}{|l|}{ Software and Algorithms } \\
\hline Comet v. 2018 & Eng et al 2013 & \\
\hline Mango & Moir et al 2018 & \\
\hline Xlink Prophet & Keller et al 2002 & \\
\hline
\end{tabular}

\section{RESOURCE AVAILABILITY}

\section{Lead contacts}

Further information and requests for resources and reagents should be directed to and will be fulfilled by the lead contacts, Rong Tian (rongtian@uw.edu) and James E. Bruce (iimbruce@uw.edu). 


\section{Materials Availability}

This study did not generate new unique reagents.

\section{Data and software availability}

Cross-linking data has been deposited at XlinkDB and is publicly available as of the date of publication. $\mathrm{DOI}$ is listed in the key resources table. The mass spectrometry proteomics data have been deposited to the ProteomeXchange Consortium via the PRIDE (Perez-Riverol et al., 2018) partner repository with the dataset identifier: PXD027757. Any additional information required to reanalyze the data reported in this paper is available from the lead contacts upon request.

\section{EXPERIMENTAL MODEL AND SUBJECT DETAILS}

\section{Animal Model}

All protocols concerning animal use were approved by the Institutional Animal Care and Use Committee at University of Washington. This study utilized 12 wild-type mice, strain C67BI6/NCrl (IMSR_CRL:27). Female and male mice have different responses to pressure overload. TAC surgery causes a mild and variable phenotype in female. Thus, only male mice were used in this study. Adult (>10 weeks old, weighing 24-26g) male mice were chosen randomly into experimental groups. Mice were housed in a vivarium with a $12 \mathrm{hr}$ light/dark cycle at $22^{\circ} \mathrm{C}$. Mice were maintained on ad libitum standard rodent diet and water.

\section{METHOD DETAILS}

Transverse aortic constriction (TAC) surgery 
Male mice aged 10-12 weeks, weighing 24-28g underwent TAC or Sham surgery as previously described (Tarnavski et al., 2004). Mice were anesthetized with $4 \%$ isoflurane and intubated with a 20 -gauge canula. Mice were ventilated at $2.5 \%$ isoflurane at 135 breaths per minute by a small animal TOPO ventilator (Kent Scientific, Torrington, CT). The aortic arch was exposed and separated from the thymus via left thoracotomy. A 27-gauge blunt needle was held near the aorta (between the brachiocephalic and left common carotid arteries). A constriction of the transverse aorta was generated by tying a 6-0 Ethilon ligature against the blunt needle. The needle was then promptly removed. The lungs were inflated and the chest was closed by $5-0$ polypropylene suture. The animal was removed from the ventilation system and given SR buprenorphine (subcutaneous, $0.5 \mathrm{mg} / \mathrm{kg}$ ) analgesic and $0.9 \%$ saline (intraperitoneal, $0.2 \mathrm{ml}$ ) for hydration after mice regained consciousness (2hrs after ventilation). Sham operated mice underwent all the same procedures as TAC, excluding ligature of the aorta. Mice were randomly assigned to TAC and Sham procedures, and researcher was blind to operation until after echocardiogram analysis was performed. Combined mortality (acute and chronic) was less than $25 \%$.

\section{Transthoracic echocardiography}

Mice were anesthetized and maintained with $0.8 \%-2 \%$ isofluorane in $95 \%$ oxygen at rates of 550-600 beats per minute as done previously(Ritterhoff et al., 2020). Trans-thoracic echocardiography was conducted 4 weeks post-TAC and Sham surgery with Vevo 2100 highfrequency, high resolution digital imaging system (VisualSonics) with a MS400 Microscan Transducer. A parasternal long-axis view was used to collect M-mode images for analysis of fractional shortening, ejection fraction, and other functional parameters. 


\section{Cross-linking of cubed heart tissue}

Mice were euthanized by cervical dislocation. The chest cavity was opened, and the heart was rapidly excised and placed into ice-cold, mitochondria isolation medium (MIM: 70mM sucrose, 220mM mannitol, 5mM MOPS, 1.6mM carnitine hydrochloride, $1 \mathrm{mM}$ EDTA, 0.025\% fatty acidfree BSA, pH 7.4 with $5 \mathrm{M} \mathrm{KOH}$ ) to flesh out blood. The aorta and atria were removed. The myocardium was weighed (heart weight). The heart was transferred to a pre-chilled $60 \mathrm{~mm}$ dry petri dish and maintained on ice. Heart tissue was finely minced with a razor blade to a homogenous tissue size distribution of approximately $1 \mathrm{~mm}^{2}$ cubes.

Heart tissue was centrifuged at $1500 \times \mathrm{g}$ for 5 min at $4^{\circ} \mathrm{C}$, and MIM was replaced with crosslinking buffer (170 mM Na2HPO4, pH 8). iqPIR (Chavez et al., 2020a) is a novel technology recently developed for quantitation of cross-linked peptide pairs using isobaric stable isotopes selectively incorporated into the PIR cross-linker, which enables $\mathrm{MS}^{2}$-based interactome quantitation in a way analogous to how TMT (Thompson et al., 2003) or iTRAQ (Ross et al.) is used for proteome quantitation. Briefly, reporter heavy $(\mathrm{RH})$ or stump heavy $(\mathrm{SH})$ iqPIR crosslinkers were applied to different samples individually first, then TAC/Sham cross-linked sample pairs were pooled together for further downstream sample processing and data acquisition.

This strategy greatly decreases sample handling errors and reduces instrument time for data acquisition. Identical cross-linked peptide pairs from TAC and sham samples have identical masses and LC retention times, yet produce different and distinct quantitative isotope signatures in $\mathrm{MS}^{2}$ spectra. The intensities of TAC- or Sham-distinct isotope labeled peptide and fragment ions in each $\mathrm{MS}^{2}$ spectrum enable relative quantitation of cross-linked peptides in TAC and Sham samples. The samples were mixed for $30 \mathrm{~min}$ at room temperature on a Thermomixer at $800 \mathrm{rpm}$. The tissue was centrifuged at $1500 \times \mathrm{g}$ for $5 \mathrm{~min}$ at $4^{\circ} \mathrm{C}$ and the 
supernatant was removed. Cross-linked tissue was then further subjected to modified mitochondrial isolation as described below.

\section{Mitochondrial isolation from cross-linked heart tissue}

Cross-linked heart tissue was washed in fresh MIM and centrifuged at $1500 \times \mathrm{g}$ for $5 \mathrm{~min}$ at $4^{\circ} \mathrm{C}$. Subsequent steps are the same as regular mitochondrial isolation protocol below. However, resulting fractions and supernatants were collected after each step. All fractions were frozen in $-80^{\circ} \mathrm{C}$ after isolation and saved for processing.

\section{Protein extraction from cross-linked mitochondria}

Frozen fractions were transferred to a stainless steel cryogrinding jar cooled to $-196^{\circ} \mathrm{C}$ with liquid nitrogen in $0.1 \mathrm{M} \mathrm{NH}_{4} \mathrm{HCO}_{3}$. The samples were cryoground for five 3 min cycles at $30 \mathrm{~Hz}$ using a Retch MM 400 mixer mill. Between cycles, the cryogrinder was cooled with liquid nitrogen between cycles. The resulting frozen powder was transferred to a falcon tube where 8M urea (in 0.1M Tris, $\mathrm{pH} 8.0$ ) was added. Samples were sonicated using a GE-130 ultasonic processor, followed by reduction of cysteine residues by incubation with 5mM Tris (2carboxyethyl) phosphine (TCEP, Fisher Scientific) for 30 min followed by alkylation with 45 min incubation with $10 \mathrm{mM}$ iodoacetamide (Fisher Scientific). Urea concentration was reduced to less than $1 \mathrm{M}$ by diluting the samples by a factor of 10 with fresh $0.1 \mathrm{M}$ Tris Buffer $(\mathrm{pH} 8.0)$. The protein concentration was measured using the Pierce Coomassie protein assay (Thermo Scientific).

\section{Protein digestion and enrichment of cross-linked peptides}


The procedures for protein digestion, desalting, and enrichment of cross-linked peptides by strong cation exchange chromatography (SCX) and biotin-capture were followed as described in a previous publication (Chavez et al., 2019b). Briefly, extracted protein were digested with trypsin (200:1 in weight ratio) overnight at $37^{\circ} \mathrm{C}$ and then quenched with acidification of digest to $\mathrm{pH} \sim 3$ by adding TFA. The digest was then desalted using Sep-Pak cartridges and fractionated on SCX using a flow rate of $1.5 \mathrm{~mL} / \mathrm{min}$ and 97.5 -min gradient with an increasing percentage of SCX solvent $\mathrm{B}$ (solvent $\mathrm{A}: 7 \mathrm{mM} \mathrm{KH}_{2} \mathrm{PO}_{4}, \mathrm{pH} 2.6,30 \%$ (vol/vol) acetonitrile and solvent B: 7 mM KH $\mathrm{PO}_{4}, \mathrm{pH}$ 2.6, $350 \mathrm{mM} \mathrm{KCl,30 \%} \mathrm{(vol/vol)} \mathrm{acetonitrile).} \mathrm{The} \mathrm{SCX} \mathrm{fractions}$ were adjusted to $\mathrm{pH} 8.0$ and then subjected to biotin capture by adding monomeric avidin UltraLink resin (Thermo Scientific) to each pooled fraction. After 3 times of washing the avidin beads with $0.1 \mathrm{M} \mathrm{NH}_{4} \mathrm{HCO}_{3}, \mathrm{pH} 8.0$, the cross-linked peptides were eluted with $70 \%$ (vol/vol) acetonitrile, $1 \%$ (vol/vol) formic acid (FA) and then dried in a speed vacuum. The dried samples were reconstituted in $0.1 \% \mathrm{FA}$ and ready for LC-MS/MS analysis.

\section{LC-MS/MS}

Cross-linked peptide samples were analyzed in technical duplicate by liquid chromatography mass spectrometry using an Easy-nLC (Thermo Scientific) coupled to a Q Exactive Plus mass spectrometer (Thermo Scientific). Each analysis used a $3 \mu \mathrm{L}$ injection of sample onto a $3 \mathrm{~cm}$ x $100 \mu \mathrm{m}$ inner diameter fused silica trap column packed with a stationary phase consisting of $5 \mu \mathrm{m}$ Reprosil C8 particles with $120 \AA$ pores (Dr. Maisch $\mathrm{GmbH}$ ) with a flow rate of $2 \mu \mathrm{L} / \mathrm{min}$ of mobile phase consisting of solvent $\mathrm{A}$ ( $\mathrm{H} 2 \mathrm{O}$ containing $0.1 \%$ formic acid) for 10 minutes. Peptides were then fractionat-ed over a $60 \mathrm{~cm}$ x $75 \mu \mathrm{m}$ inner diameter fused silica analytical column packed with $5 \mu \mathrm{m}$ Reprosil C8 particles with $120 \AA$ p pores by applying a linear gradient 
from $95 \%$ solvent $A, 5 \%$ solvent $B$ (acetonitrile containing $0.1 \%$ formic acid) to $60 \%$ solvent $A$, $40 \%$ solvent B over either 120 or 240 minutes at a flow rate of $300 \mathrm{~nL} / \mathrm{min}$. Eluting peptide ions were ionized by electrospray ionization by applying a positive $2.2 \mathrm{kV}$ potential to a laser pulled spray tip at the end of the analytical column. The mass spectrometer was operated using a top five data dependent acquisition method with a resolving power setting of 70,000 for $\mathrm{MS}^{1}$ and $\mathrm{MS}^{2}$ scans. Additional settings include an AGC target value of $1 \mathrm{e} 6$ with a maximum ion time of $100 \mathrm{~ms}$ for the $\mathrm{MS}^{1}$ scans and an AGC value of $5 \mathrm{e} 4$ with a maximum ion time of $300 \mathrm{~ms}$ for the $\mathrm{MS}^{2}$ scans. Charge state exclusion parameters were set to only allow ions with charge states from $4+$ to $7+$ to be selected for $\mathrm{MS}^{2}$. Ions selected for $\mathrm{MS}^{2}$ were isolated with a $3 \mathrm{~m} / \mathrm{z}$ window and fragmented by HCD using a normalized collision energy setting of 30 . Ions for which $\mathrm{MS}^{2}$ was performed were then dynamically excluded from further selection for $\mathrm{MS}^{2}$ for $30 \mathrm{~s}$.

\section{Heat Map Analysis}

Perseus (version 1.6.15.0) (Tyanova et al., 2016) was used for statistical data analysis of cross-link quantitation ratios. Data was first filtered based on $70 \%$ of total valid values across all biological replicates and then applied with T-test using Benjamini-Hochberg FDR at 0.05. T-test q-values less than 0.05 were considered significant. Heatmaps were generated using NG-CHM GUI software (version 2.20.0) (Broom et al., 2017) with Euclidean distance and complete linkage selected for both row and column hierarchical clustering.

\section{Mitochondrial Isolation from cardiac tissue}

Hearts were excised from mice, the aortas and atria were removed. Heart tissues were rinsed 
briefly in ice-cold mitochondria isolation medium (70mM sucrose, $220 \mathrm{mM}$ mannitol, $5 \mathrm{mM}$ MOPS, 1.6mM carnitine hydrochloride, 1mM EDTA, $0.025 \%$ fatty acid-free BSA, pH 7.4 with $5 \mathrm{M} \mathrm{KOH}$ ) with the addition of $2 \mathrm{mM}$ taurine, to remove residual blood. Tissues were minced on ice and resuspended in fresh MIM, followed by trypsin digestion (10ug/ml) and incubated on ice for $10 \mathrm{~min}$. Trypsin digestion was stopped by the addition of trypsin inhibitor $(0.5 \mathrm{mg} / \mathrm{ml})$ and additional BSA (1 mg/ml) to MIM. The suspension was centrifuged for $1 \mathrm{~min}$ at $1,500 \times \mathrm{g}$ at $4^{\circ} \mathrm{C}$, and the supernatant was discarded. The tissue pellets were resuspended in fresh MIM containing $1 \mathrm{mg} / \mathrm{ml}$ BSA, and transferred to a Teflon-glass tube and homogenized on ice with a Teflon pestle. The homogenates were centrifuged for $10 \mathrm{~min}$ at $800 \mathrm{xg}$ at $4^{\circ} \mathrm{C}$. The supernatants were collected and centrifuged for $10 \mathrm{~min}$ at $8,000 \times \mathrm{g}$ at $4^{\circ} \mathrm{C}$. The supernatant was discarded and the mitochondrial pellets were resuspended in MIM to wash. The resuspension was centrifuged for $10 \mathrm{~min}$ at $8,000 \times \mathrm{g}$ at $4^{\circ} \mathrm{C}$, and the supernatant discarded. The mitochondrial pellet was resuspended to a concentrated volume for protein quantification by BCA Assay (Pierce).

\section{Mitochondrial respiration using Seahorse XFe24 Analyzer}

The oxygen consumption rate (OCR) of isolated cardiac mitochondria was measured with an XF24e Flux Analyzer (Seahorse Bioscience, Agilent) based off the manufacturer's protocol(Rogers et al., 2011). The Seahorse utility plate was hydrated with calibration buffer and incubated overnight at $37^{\circ} \mathrm{C}$ prior to respiration experiments. The mitochondria were seeded in 24-well microplates at 1-2ug protein per well in 1x mitochondrial assay solution (70mM sucrose, 220mM mannitol, $10 \mathrm{mM} \mathrm{KH}_{2} \mathrm{PO}_{4}, 5 \mathrm{mM} \mathrm{MgCl}_{2}, 2 \mathrm{mM}$ HEPES, $1 \mathrm{mM}$ EGTA, $0.2 \%$ BSA, $\mathrm{pH} 7.35$ at room temperature) containing substrates and centrifuged for 2,000 $\times \mathrm{g}$ 
for 20 min at $4^{\circ} \mathrm{C}$. Respiration buffer was added to each well to a final volume of $500 u$ ul, then the plate was placed in a non- $\mathrm{CO} 2$ incubator for $10 \mathrm{~min}$ at $37^{\circ} \mathrm{C}$. The plate was incubated the Seahorse Analyzer and a baseline oxygen consumption rate (OCR) was measured. Sequential injections of inhibitors (final concentration 4mM ADP, $2.5 \mathrm{ug} / \mathrm{mL}$ oligomycin, $4 \mathrm{uM}$ carbonyl cyanide-4-(trifluoromethoxy)phenylhydrazone, and 4uM antimycin A) were added into each well and changes in OCR were measured. For Complex I-supported respiration, 10mM pyruvate and $2 \mathrm{mM}$ malate were given to mitochondria seeded at $2 \mathrm{ug} /$ well.

\section{Molecular docking calculation}

Crystal structures of tetrameric of human SCOT1 (PDB: 3DLX) were subjected to rigid body molecular docking using online platform, SymmDock (Schneidman-Duhovny et al., 2005). Without applying distance constraints, the ten highest scoring solutions were analyzed with the biomolecular visualization tool, Pymol. Residues and sidechains surrounding K296 (human 284-286 and 297-299) are shown as yellow spheres because residues 296-296 are unresolved in all solved structures. The putative interface between SCOT1 structures consistently puts regions surrounding K296 of opposite tetramers nearby (within $42 \AA$ distance in 6 of the top 10 solutions).

\section{Cytochrome C Oxidase and Citrate Synthase activity from heart tissue homogenate}

Citrate synthase (CS) and Cytochrome C Oxidase (COX) activities were determined in heart tissue homogenates at $30^{\circ} \mathrm{C}$ using a protocol adapted from previously published methods (Spinazzi et al., 2012). Briefly, for CS activity frozen cardiac tissue (25mg) was homogenized at $4^{\circ} \mathrm{C}$ in CelLytic ${ }^{\mathrm{TM}} \mathrm{MT}$ Cell Lysis Reagent (C3228) and then incubated on ice for 30 minutes. 
The samples were then centrifuged at $10,000 \times \mathrm{g}$ for 10 minutes at $4^{\circ} \mathrm{C}$ and protein concentration determined. CS activity was analyzed in $100 \mathrm{mM}$ Tris buffer containing $0.1 \%$ Triton X-100, pH 8.0 as previously described (Spinazzi et al., 2012).

For COX activity, $25 \mathrm{mg}$ of cardiac tissue was homogenized at $4^{\circ} \mathrm{C}$ in $50 \mathrm{mM}$ potassium phosphate buffer containing $1 \mathrm{mM}$ EDTA, and $0.1 \%$ Triton X-100 $(\mathrm{pH} 7.4$; final concentration, 5 $\mathrm{mg}$ tissue $/ \mathrm{mL}$ ). The samples were incubated on ice for 30 minutes followed by centrifugation at $10,000 \times \mathrm{g}$ for 10 minutes at $4^{\circ} \mathrm{C}$. COX activity was analyzed in $100 \mathrm{mM}$ potassium phosphate buffer as previously described (Spinazzi et al., 2012).

\section{QUANTIFICATION AND STATISTICAL ANALYSIS}

\section{Assessment of cardiac and mitochondrial function}

The numbers of independent experiments are specified in the relevant figure legends. Data are expressed as the mean \pm standard error of the mean (SEM). Statistical comparisons between two groups were conducted by unpaired, two-tailed Student's $t$-test. $P<0.05$ was considered to be statistically significant. Statistical analysis was performed with Prism 9.0 software (GraphPad).

\section{LC-MS/MS data analysis}

The LC-MS/MS results were analyzed for identification and quantitation of iqPIR cross-linked peptide pairs as previously described (Chavez et al., 2020a). Briefly, LC-MS/MS data raw files were converted to .mzXML format using the ReADW tool in the Trans Proteomic Pipeline software suite (Keller et al., 2005). Mango (Mohr et al., 2018) was used to search for PIR mass relationships and Comet (Chavez et al., 2013) was used to search the mzXML files against the 
mouse Mitocarta 2 database (Calvo et al., 2015) consisted of both forward and reverse protein sequences $(2,084$ total sequences). The resulting pepXML files were then analyzed with XLinkProphet (Keller et al., 2018) and filtered for estimated 1\% FDR at the non-redundant cross-link level. For dead-end peptides, PeptideProphet (Keller et al., 2002) was applied to filter Comet search results at $<1 \%$ FDR. All cross-links passing the threshold were used for quantitation. Light $(\mathrm{SH})$ and heavy $(\mathrm{RH})$ isotope peptide precursors and their fragment peaks containing the stump group were deconvoluted and their peak intensities were extracted to calculate Log2 ratios. The final ratio for each cross-linked peptide pair is the normalized mean value from all contributing ion ratios to the same cross-link from different charge states, different scans, and separate replicate runs with outliers removed, where normalization is achieved by subtracting from each cross-link Log2 ratio the median value of all cross-link Log2 ratios. Finally, the cross-link Log2 ratio p-value reflecting its likelihood of being 0 is calculated based on a statistical t-test using the Log2 ratio mean value, standard deviation, and number of contributing quantified ions. Protein quantitation was estimated by combining together normalized Log2 ratios of all intra-protein cross-links and dead-end peptides corresponding to the protein. The mean Log2 ratio of all contributing ratios (intra-protein cross-link and deadend) was used after excluding outliers. 